\title{
Corticocortical and Thalamocortical Changes in Functional Connectivity and White Matter Structural Integrity after Reward-Guided Learning of Visuospatial Discriminations in Rhesus Monkeys
}

\author{
${ }^{D}$ Vassilis Pelekanos, ${ }^{1}{ }^{\circledR}$ Elsie Premereur, ${ }^{2}{ }^{-}$Daniel J. Mitchell, ${ }^{3}$ Subhojit Chakraborty, ${ }^{4}$ Stuart Mason, ${ }^{1}$ \\ ${ }^{-}$Andy C.H. Lee, ${ }^{5,6}$ and ${ }^{\circledR}$ Anna S. Mitchell ${ }^{1}$ \\ ${ }^{1}$ Department of Experimental Psychology, University of Oxford, Oxford OX1 3SR, United Kingdom, ${ }^{2}$ Laboratory for Neuro- and Psychophysiology, KU \\ Leuven, 3000 Leuven, Belgium, ${ }^{3} \mathrm{MRC}$ Cognition and Brain Sciences Unit, University of Cambridge, Cambridge CB2 7EF, United Kingdom, ${ }^{4}$ Department \\ of Neuroinflammation, Queen Square Multiple Sclerosis Centre, Institute of Neurology, University College London, London WC1N 3BG, United \\ Kingdom, ${ }^{5}$ Department of Psychology (Scarborough), University of Toronto, Toronto, Ontario M1C 1A4, Canada, and ${ }^{6}$ Rotman Research Institute, \\ Baycrest Centre, Toronto, Ontario M6A 2E1, Canada
}

The frontal cortex and temporal lobes together regulate complex learning and memory capabilities. Here, we collected resting-state functional and diffusion-weighted MRI data before and after male rhesus macaque monkeys received extensive training to learn novel visuospatial discriminations (reward-guided learning). We found functional connectivity changes in orbitofrontal, ventromedial prefrontal, inferotemporal, entorhinal, retrosplenial, and anterior cingulate cortices, the subicular complex, and the dorsal, medial thalamus. These corticocortical and thalamocortical changes in functional connectivity were accompanied by related white matter structural alterations in the uncinate fasciculus, fornix, and ventral prefrontal tract: tracts that connect (sub)cortical networks and are implicated in learning and memory processes in monkeys and humans. After the well-trained monkeys received fornix transection, they were impaired in learning new visuospatial discriminations. In addition, the functional connectivity profile that was observed after the training was altered. These changes were accompanied by white matter changes in the ventral prefrontal tract, although the integrity of the uncinate fasciculus remained unchanged. Our experiments highlight the importance of different communication relayed among corticocortical and thalamocortical circuitry for the ability to learn new visuospatial associations (learning-to-learn) and to make reward-guided decisions.

Key words: cingulate cortex; learning; mediodorsal thalamus; orbitofrontal cortex; reward; uncinate fasciculus

Significance Statement

Frontal neural networks and the temporal lobes contribute to reward-guided learning in mammals. Here, we provide novel insight by showing that specific corticocortical and thalamocortical functional connectivity is altered after rhesus monkeys received extensive training to learn novel visuospatial discriminations. Contiguous white matter fiber pathways linking these gray matter structures, namely, the uncinate fasciculus, fornix, and ventral prefrontal tract, showed structural changes after completing training in the visuospatial task. Additionally, different patterns of functional and structural connectivity are reported after removal of subcortical connections within the extended hippocampal system, via fornix transection. These results highlight the importance of both corticocortical and thalamocortical interactions in reward-guided learning in the normal brain and identify brain structures important for memory capabilities after injury.

Received Feb. 26, 2020; revised June 30, 2020; accepted July 25, 2020

Author contributions: V.P., E.P., and D.J.M. contributed unpublished reagents/analytic tools; V.P., E.P., D.J.M., A.C.H.L., and A.S.M. analyzed data; V.P. and A.S.M. wrote the first draft of the paper; V.P., E.P., D.J.M., S.M., S.C., A.C.H.L., and A.S.M. edited the paper; V.P., A.C.H.L., and A.S.M. wrote the paper; S.M., S.C., and A.S.M. performed research; A.C.H.L. and A.S.M. designed research.

The authors declare no competing financial interests.
This work was supported by Medical Research Council UK G0800329 and Wellcome Trust WT 110157/Z/15/Z to A.S.M. and WT 082315 to A.C.H.L. We thank David Gaffan for discussions on the design of the monkey training task; Kentaro Miyamoto for helpful discussions on MRI data analysis; and Andrew Bell and Vasilis Karlaftis for helpful discussions on DTI analysis.

Correspondence should be addressed to Anna S. Mitchell at anna.mitchell@psy.ox.ac.uk.

https://doi.org/10.1523/JNEUROSCI.0364-20.2020

Copyright $\odot 2020$ the authors 


\section{Introduction}

The ability to learn visuospatial information is key to survival and facilitates optimal decision-making. Yet, understanding the neural mechanisms that support this learning remains a critical question in neuroscience. The orbitofrontal cortex (OFC) and ventromedial prefrontal cortex (vmPFC) contribute to cognitive control during learning and decision-making (Eichenbaum, 2017; Murray and Rudebeck, 2018), and inferotemporal cortex contributes to visually guided discriminations (Ettlinger, 1959; Fahy et al., 1993; Buckley and Gaffan, 1998; Ungerleider and Mishkin, 1982; Lee et al., 2005). Reinforcement meta-learning (learning-to-learn), that is, acquiring and retaining the use of the same rule on different occasions, is linked to optimal frontal network functioning and dopamine (Wang et al., 2018). The frontal cortex and the temporal lobes are anatomically connected via white matter fiber tracts, such as the uncinate fasciculus (UF) and the fornix (Poletti and Creswell, 1977; Ungerleider et al., 1989; Schmahmann and Pandya, 2006; Saunders and Aggleton, 2007; Insausti and Amaral, 2008). In humans, diffusion-weighted MRI highlights that the UF is involved in learning complex visuospatial tasks (Metzler-Baddeley et al., 2011) and in episodic memory function (Metzler-Baddeley et al., 2012). There are also marked changes in the UF in patients with dementia (Von Der Heide et al., 2013). Additionally, the fornix is associated with recollection and recall in MRI studies (Rudebeck et al., 2009; Metzler-Baddeley et al., 2011) and has shown changes in patients with hippocampal lesions (Henson et al., 2016). Interestingly, causal evidence from humans and nonhuman primates further shows that transecting the UF or the fornix impairs cognitive performance in learning and memory tasks (D’Esposito et al., 1995; Aggleton et al., 2000; Gaffan, 2002; Browning and Gaffan, 2008; Mitchell et al., 2008; Tsivilis et al., 2008; Kwok et al., 2015). However, it remains unclear how learning new visuospatial information alters (sub)cortical connectivity, and how, following the loss of subcortical inputs to the cortex, this connectivity is rearranged. In this study, we combined visuospatial training on a complex cognitive task and neuroimaging in nonhuman primates to investigate learning-induced changes in (sub)cortical connectivity. We used resting-state functional MRI (rsfMRI), to explore longitudinal changes in functional connectivity, and diffusion tensor imaging (DTI) to detect changes in white matter structural connectivity. We collected our rsfMRI and DTI data before and after our monkeys learned a complex visuospatial discrimination task that involved several months of training (Gaffan, 1994; Aggleton and Brown, 2006). Our first hypothesis was that, after learning the task, brain areas linked with rapid associative learning of new, complex visuospatial information, such as the inferotemporal cortex, OFC, and vmPFC, the subicular complex, and dorsal medial thalamus, would show changes in functional and structural connectivity.

In the second part of our study, we sought to determine the consequences of fornix lesion on the trained monkeys' brain connectivity. We targeted the fornix, a major subcortical white matter tract that connects the frontal cortex and extended hippocampal system with the medial diencephalon (Krayniak et al., 1979; Aggleton et al., 1986, 2005; Xiao et al., 2009).

Consistent with previous results, frontotemporal structures changed their functional connectivity and white matter microstructural integrity after learning to learn our visuospatial task, with these changes linked to specific OFC, vmPFC, subicular complex, medial prefrontal, and inferotemporal lobe interactions. Remarkably, temporal lobe functional connectivity to dorsal medial thalamus were also altered after learning. Further, the fornix transections impaired the monkeys' ability to learn new visuospatial discriminations, accompanied by marked changes to the training-related connectivity profiles. Overall, our study provides novel understanding of the involvement of corticocortical and corticothalamic connectivity in visuospatial learning, and memory-guided decision-making. Given the evidence for extensive dorsal, medial thalamic alterations in the dementias and schizophrenia, and subicular complex alterations in Alzheimer's disease (Braak and Braak, 1991; Aggleton et al., 2016; Huang et al., 2019; Bocchetta et al., 2020), our findings may also provide disease-relevant insights to help unravel the links between (sub) cortical frontotemporal brain connectivity, and learning and memory capabilities.

\section{Materials and Methods}

Subjects and housing

All experimental procedures were performed in compliance with the United Kingdom Animals (Scientific Procedures) Act of 1986. A Home Office (UK) Project License obtained after review by the University of Oxford Animal Care and Ethical Review committee licensed all procedures. The housing and husbandry complied with the ARRIVE guidelines of the European Directive (2010/63/EU) for the care and use of laboratory animals.

We used 8 male rhesus macaque monkeys (Macaca mulatta) aged 5 years at the beginning of the study (mean weight $=5.06 \mathrm{~kg}, \mathrm{SD}=0.4$ ). The monkeys in the Experimental group $(n=4)$ were housed in one group, whereas the monkeys in the Control group $(n=4)$ were housed together in a separate group. All monkeys received several MRI scans consisting of T1 structural (MPRAGE) scans, resting-state functional scans, and diffusion-weighted scans, at key milestones in visuospatial training during the course of the experiments, under general anesthesia.

\section{Task training and experimental design}

We collected MRI data for an Experimental group and a Control group of monkeys, at around the same time over the course of the experiments. The Experimental group received extensive training on a complex visuospatial task for an average of 17 months to master the rapid learning of new visuospatial information during each testing session. We used a variant of the object-in-place discrimination (OIP) task (Gaffan, 1994) that assesses rapid learning of visuospatial discriminations in nonhuman primates (Murray and Wise, 2010) and humans (Aggleton et al., 2000). The task was adapted so that it required a longer time to learn and attain a consistent learning criterion.

Experimental group. The computer-controlled test apparatus was identical to that described previously (Mitchell et al., 2007). Briefly, the 4 monkeys who trained in the visuospatial learning task sat in a transport box fixed to the front of a large touch-sensitive color monitor that displayed the stimuli for all experiments. Monkeys reached with their hand to respond on the touchscreen and collect their food reward pellets (190 mg banana-flavored pellets) that were automatically dispensed from a hopper by the computer. Monkeys were monitored remotely via closed-circuit cameras and display monitors throughout each session.

After recovery from their first MRI scan, the Experimental group went through task training involving operant learning, associative reinforcement learning, and meta-reinforcement learning to optimize their rewards. Initially, the monkeys were acclimatized to the transport box and computer touchscreen setup using standardized training procedures (Mason et al., 2019). Then the monkeys learned that, if they touched the touchscreen, they would receive a reward (operant learning). Then they learned that touching certain objects presented on the touchscreen gave them a reward (associative reinforcement learning). Next, they moved onto our adapted version of the OIP task. For each trial of this task, two identical bull's-eye "objects" (a white round circle, $5 \mathrm{~cm}$ in diameter; with a smaller black circle, $1 \mathrm{~cm}$ in diameter; in the middle, a bull's-eye; see Fig. 1) were presented at different locations within a trial-unique colorful background. The background filled the whole touchscreen and consisted of randomly generated shapes in randomly generated different colors (see Fig. 1A,B). The monkeys were required to learn, via trial and 
A

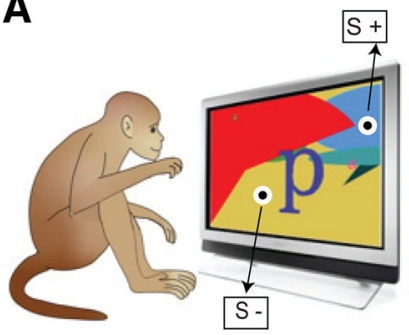

B
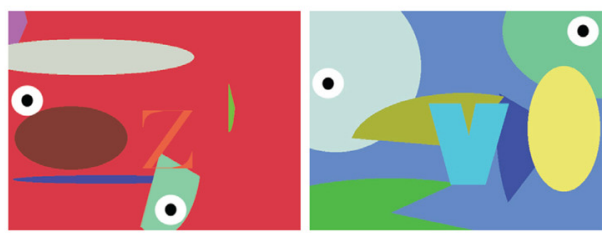

C

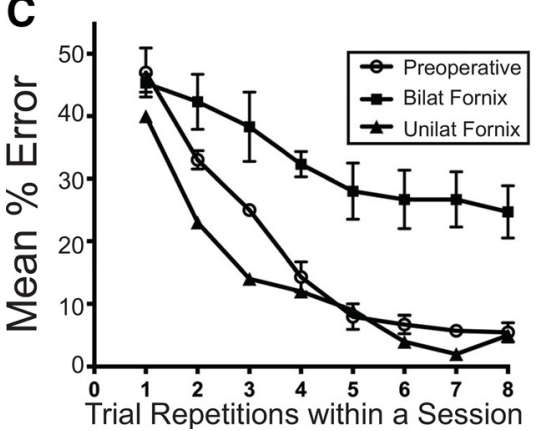

D

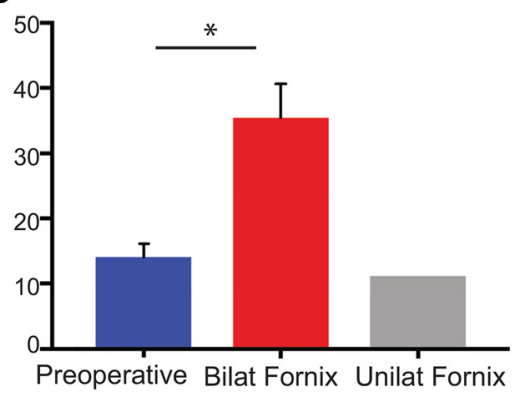

Figure 1. The visuospatial task and the monkeys' preoperative and postoperative learning performance. $\boldsymbol{A}$, The visuospatial task. On each training session, monkeys in the Experimental group had to learn, for each trial, which one of two identical objects (black-white round circles) embedded within a unique complex background presented on a touchscreen computer led to receiving a food reward (correct; $S_{+}+$) and which one was incorrect (no reward; $S_{-}$) (see Materials and Methods). $\boldsymbol{B}$, Two example trials. $\boldsymbol{C}$, Mean percent errors across trial repetitions within each session for the preoperative (open circles) and postoperative performance tests in the 3 monkeys with bilateral fornix transection (black squares) and the 1 monkey with unilateral fornix damage (black triangles). D, Overall mean percent errors during the preoperative and postoperative performance tests. Error bars indicate SEM. ${ }^{*} p<0.05$.

error, that for each unique background, touching one bull's-eye object would lead to a reward, whereas touching the other would not. In each daily session, there were 10 unique trials, each presented 8 times in total. New trials were created for each daily session, with identical bull's-eyes being placed randomly at different locations within the unique background.

With training, the monkeys used their previous knowledge acquired about how to solve the task to optimize their rewards within each session. Over time, the monkeys completed the task faster and learned to maximize their rewards (for data, see Results) to a criterion of no more than 12 errors on average per session across 2 consecutive weeks of training. All monkeys in our Experimental group reached a consistent level of learning within 16-18 months of training. Upon attaining this criterion, the monkeys underwent another MRI scan. Then after several days of recovery, they completed a preoperative performance test. The preoperative test consisted of the monkeys working for $2 \mathrm{~d}$ completing 5 trials repeated 8 times, and then working for $11 \mathrm{~d}$ completing 10 trials repeated 8 times. Data were analyzed from the last $10 \mathrm{~d}$ of the test. The postoperative test was identical to the preoperative test and was completed after at least 2 weeks recovery from the surgery.

The first scan of monkeys in the Experimental group took place before their training began, that is, at Time Point 1 (Exp_tp1). The second scan (Exp_tp2) took place after training, on attaining the consistent learning criterion. After Exp_tp2, the Experimental group monkeys underwent neurosurgery to receive a selective fornix transection. After postoperative recovery of 2 weeks, these monkeys completed a postoperative performance test, and then received their final scan (Postop).

Control group. The age- and cohort-matched monkeys in the Control group were also scanned at Time Point 1 (Con_tp1). This was conducted within a 2 month window from the first scan of the Experimental group. Then the Control group monkeys were scanned at Time Point 2 (Con_tp2). Again, this was conducted within roughly a 2 month window from the second scan of the Experimental group.
In contrast to the complex cognitive training of the Experimental group, the Control group completed a passive visual fixation task. The task, stimuli, and training protocol of our control monkeys are described in detail previously (Pelekanos et al., 2020). Briefly, control monkeys were trained to fixate a cue that appeared in the center of a computer screen. Forty-eight images of faces, body parts, objects, and places were presented, during training in each session, in the center of the screen. For each session, each image was presented once, in randomized order, and monkeys received a smoothie reward for maintaining fixation within a $5^{\circ} \times 5^{\circ}$ rectangular frame appearing in the center of each image. The 4 Control group monkeys received a headpost implanted onto the skull under general anesthesia between tp1 and tp 2 . We reasoned that the training of the Control group on this unrelated, nonmnemonic task rendered these monkeys a suitable baseline control for the monkeys in the Experimental group.

\section{Imaging protocols and data acquisition}

We measured brain activity using rsfMRI and DTI, which is a special diffusion-weighted MRI technique. To evaluate functional connectivity, we calculated the correlation strength in the spontaneous rsfMRI BOLD signal between pairs of gray matter areas. Specifically, we analyzed signal fluctuations to assess whether the functional coupling between pairs of gray matter areas changed after learning in the OIP task and after fornix lesions. Our analysis focused on specific areas in the frontal and temporal lobes, cingulate cortex, and dorsal midline thalamus, which have been strongly associated with learning and memory. To evaluate structural connectivity (the connectivity of brain areas anatomically linked to one another), we measured the DTI-derived index of fractional anisotropy (FA) that reflects white matter microstructural integrity (Beaulieu et al., 2002). Our DTI analysis focused on bilateral masks of the fornix, the UF, and the ventral prefrontal (VP) white matter (for full details, see White matter tracts of interest (TOIs)). Using DTI, we investigated structural connectivity, that is, the connectivity of brain areas that are anatomically linked to one another. Diffusion-weighted imaging is sensitive to the random translational motion of water molecules, producing MRI-based maps of this physical diffusion process in brain tissue (Le Bihan, 2003). The diffusion of water molecules is hindered by cell membranes and/or fibers, and signal in diffusion-weighted images is modulated by the density of white matter fiber tracts, which are indirectly visualized.

All data were collected using a horizontal 3T MRI scanner and a custom-made four-channel phased-array receiver coil, together with a radial transmission coil (Windmiller Kolster Scientific). The anesthetized monkeys were placed in the scanner in a feet-first prone "sphinx" position in an MRI-compatible stereotaxic frame (Crist Instrument). For sedation procedures and the maintenance of general anesthesia, we followed previously described procedures (Mars et al., 2011; Mitchell et al., 2016). Briefly, anesthesia was induced using intramuscular injection of ketamine $(10 \mathrm{mg} / \mathrm{kg})$ combined with either xylazine $(0.125-0.25 \mathrm{mg} / \mathrm{kg})$ or midazolam $(0.1 \mathrm{mg} / \mathrm{kg})$ and buprenorphine $(0.01 \mathrm{mg} / \mathrm{kg})$. An intravenous cannula was used for delivery of fluids (warmed sterile saline drip, $5 \mathrm{ml} / \mathrm{h} / \mathrm{kg}$ ) throughout the scan. Monkeys were intubated, ventilated with intermittent positive pressure to ensure a constant respiration rate during the functional scan, and placed on isoflurane anesthesia. The lowest possible concentration of isoflurane gas (mixed with medical oxygen) was used to maintain anesthesia, in accordance with veterinary instructions. Across all monkeys, in both groups and all scans, the inspired and expired isoflurane concentrations were consistently in the range of $1.2 \%$ $1.8 \%$ (mean $1.48 \%$ ) and $1.1 \%-1.6 \%$ (mean $1.38 \%$ ), with the only exceptions being the inspired and expired isoflurane concentrations for 1 
monkey in the Experimental group at the Expt_tp2 (2.1\% and 2\%, respectively) and Postop ( $2.3 \%$ and $2.1 \%$, respectively) scans. Under these conditions, resting-state networks are reliably observed (Vincent et al., 2007; Mars et al., 2011; Hutchison et al., 2014; Noonan et al., 2014). Normal body temperature was maintained throughout the scan using blankets, bubble wrap, and heated wheat bags. Respiration rate, inspired and expired $\mathrm{CO}_{2}$, and inspired and expired isoflurane concentration were monitored and recorded using VitalMonitor software (Vetronic Services).

Our MRI protocols were identical to those used by other primate researchers performing MRI scanning at University of Oxford (Milham et al., 2018). For each scan, we first collected the T1-weighted, high-resolution ( $0.5 \mathrm{~mm}$ isotropic voxels) structural images using an MPRAGE ( $\mathrm{TR}=2.5 \mathrm{~s}$, echo time $=4.01 \mathrm{~ms}, 3-5$ averages) sequence. This was followed by the fMRI data acquisition, in which we used an EPI sequence, and collected a total of $\sim 1600$ volumes per monkey. Imaging parameters included the following: voxel size $=2 \mathrm{~mm}$ isotropic, $\mathrm{TR}=2 \mathrm{~s}, 36$ slices, echo time $=19 \mathrm{~ms}$, flip angle $=78^{\circ}$. Finally, for the diffusion-weighted data acquisition, we used an EPI sequence with the following imaging parameters: voxel size $=1 \mathrm{~mm}$ isotropic, $\mathrm{b}$ values $=1$ and 1000, 60 isotropically distributed diffusion-encoding directions, $\mathrm{TR}=8.3 \mathrm{~s}$, and echo time $=102 \mathrm{~ms}$, with alternative phase-encoding directions (anterior-posterior, posterior-anterior). We collected six averages (all 60 diffusion-encoding directions and $11 \mathrm{~b} 0$ images in each average) in two alternating phase-encoding directions within a single diffusion-weighted scan session.

\section{rsfMRI data preprocessing}

Functional data were analyzed using the pipeline described in detail previously (Mitchell et al., 2016; Ainsworth et al., 2018). Briefly, structural volumes for each monkey were coregistered to the 112 rhesus macaque standard anatomic template (in the space of the atlas) (Saleem and Logothetis, 2006), and segmented into gray matter, white matter, and CSF tissue classes (McLaren et al., 2009). Functional volumes (excluding the 6 volumes acquired first in a scan) were used to estimate movement parameters, and were coregistered to the structural volumes for each monkey. Functional data were spatially smoothed using a Gaussian kernel with $3 \mathrm{~mm}$ FWHM. Gray matter ROI masks were defined, per monkey, as voxels with gray matter probability $>0.9$.

\section{rsfMRI ROIs}

Our ROIs in the resting-state experiment consisted of a network of gray matter areas that have previously been shown to contribute to learning and visuospatial memory. Specifically, we included the ventrolateral $\mathrm{PFC}$, vmPFC, and OFC, in particular, the lateral and medial portions of area $11(11 \mathrm{l}, 11 \mathrm{~m})$, area $12(12 \mathrm{l}, 12 \mathrm{~m})$, and area $13(13 \mathrm{l}, 13 \mathrm{~m})$, the orbital area $12(12 \mathrm{o})$, the rostral area $14(\mathrm{r})$, and the subgenual area 25 , and dorsal ACC (areas 24ab and 32) (Aggleton et al., 2015). Our ROIs additionally included parts of the extended hippocampal system (Papez circuit), including the subicular complex [parasubiculum, prosubiculum, presubiculum, subiculum (PaS, ProS, PreS, and Sub, respectively, in Fig. 2)] and retrosplenial cortex (29a, 29ac, 29d, and 30). We also included the perirhinal cortex (areas 35 and 36), entorhinal cortex (area 28), and area TE on the lateral surface of the inferotemporal cortex. Finally, we also included a dorsal medial thalamic (DM_Th) mask that was hand-drawn to include dorsal midline and limbic thalamic structures, including the mediodorsal thalamus, dorsal midline thalamic nuclei, and midline parts of the anterior thalamic nuclei. Mediodorsal and anterior thalamic nuclei have been shown to contribute to rapid learning of new visuospatial discriminations in monkeys (Parker and Gaffan, 1997; Mitchell et al., 2007; Mitchell and Gaffan, 2008; Browning et al., 2015). Anatomical gray matter masks for both hemispheres were delineated according to the macaque cortical parcellations (Van Essen et al., 2012).

\section{Analysis of changes in functional connectivity}

Covariates were constructed to model physiological confounds by extracting up to six principal components of the times series from the white matter tissue mask, to explain $99 \%$ of variance (Behzadi et al., 2007); their temporal derivatives were also added, plus a similar set of covariates derived from the CSF mask. A covariate to model motion confounds was defined as the time course of average displacement over the expected brain volume (approximated as a $40 \mathrm{~mm}$ radius sphere) (Jenkinson, 1999). The temporal derivative of the motion vector was additionally included, along with the element-wise squares of both these vectors. A temporal bandpass filter $(0.0025-0.05 \mathrm{~Hz})$ was implemented using additional discrete cosine transform covariates. After projecting these covariates from the BOLD signal time series of each voxel, functional connectivity was estimated as bivariate correlations between the mean time series from pairs of gray matter ROIs. Fisher $z$ transform of the correlation coefficients ( $r$ to $z$ ) was then applied. To assess the training-related changes in functional connectivity, for every ROI-ROI pair, and each subject, we calculated the difference in correlation strength (i.e., in functional connectivity) between the two time points (for the Experimental and the Control groups (see Fig. $2 A$ and $B$ respectively). Next, we calculated the average (across subjects) differences in functional connectivity (between both time points) between the Experimental and Control groups; that is, we tested for the interaction [Exp_tp2 Exp_tp1] - [Con_tp2 - Con_tp1] (see Fig. 2C). To assess statistical significance of these differences, we performed a permutation test in which the subjects (and sessions) labels for each ROI-ROI pair were randomly shuffled. For each permutation, the absolute value of the difference between (the randomly shuffled) sessions was calculated. ROI-ROI pairs for which the real absolute difference was larger than the 95th percentile (one-tailed) of the null distribution (differences calculated after shuffling) are indicated with one asterisk in Figure 2. Two asterisks indicate ROI-ROI pairs for which the real difference was larger than the 95th percentile (one-tailed) but FDR-corrected (Benjamini and Hochberg, 1995; Genovese et al., 2002) for multiple comparisons.

To assess the lesion-related changes in functional connectivity, we calculated the average differences in functional connectivity between the Postop session and the Exp_tp2 session (see Fig. 2D). Significance was assessed following the same permutation pipeline.

\section{Analysis of changes in structural connectivity}

Diffusion-weighted imaging data were corrected for susceptibility field distortions along the phase-encoding direction offline, using Top-Up (Andersson et al., 2003; Smith et al., 2004), an EPI image distortion correction tool, which is part of the FSL suite. We further applied orientation correction, skull-stripping, and mask editing before applying DTI. We used DTIFit, an FSL tool to fit a diffusion tensor model at each voxel in our diffusion-weighted data.

We considered FA, which is a common DTI-derived measure, thought to quantify microstructural integrity and reflect properties, such as the diameter, density, and myelination of a fiber tract (Beaulieu et al., 2002). To test for white matter differences between two conditions (e.g., tp2 vs tp1 for the Experimental group), we compared FA maps in major white matter tracts implicated in visuospatial memory (see below). For any given test, we performed voxelwise statistical analysis on the FA maps using tract-based spatial statistics (TBSS) (Smith et al., 2006). First, we registered the FA maps of each subject to a standard anatomic template (MACAQUE-F99) (Van Essen, 2002; Van Essen et al., 2012) using affine transformation. The resulting images were then merged into a single $4 \mathrm{D}$ image file, the mean of which was used (threshold with FA $>0.2$ ) to produce an alignment-invariant, test-specific, tract representation mask (the "skeleton"). The 4D image file was then used to project the individual subjects FA maps onto the skeleton, resulting in a final file consisting of all tracts common to the given group of subjects (Smith et al., 2006).

\section{White matter tracts of interest (TOIs)}

The TBSS analysis described above was conducted within white matter TOIs, namely, the fornix, the UF, and the VP (see Fig. 4). The fornix connects the extended hippocampal system to the PFC (Poletti and Creswell, 1977), while the UF links the anterior temporal lobe to the vmPFC (Ungerleider et al., 1989; Schmahmann and Pandya, 2006). The VP white matter in the mask we used lies inferior and slightly medial to the anterior corona radiata (Zakszewski et al., 2014). The VP connects ventral PFC structures, and contributes fibers to the corona radiata that connects PFC to the dorsal, medial thalamus, in both macaques and 

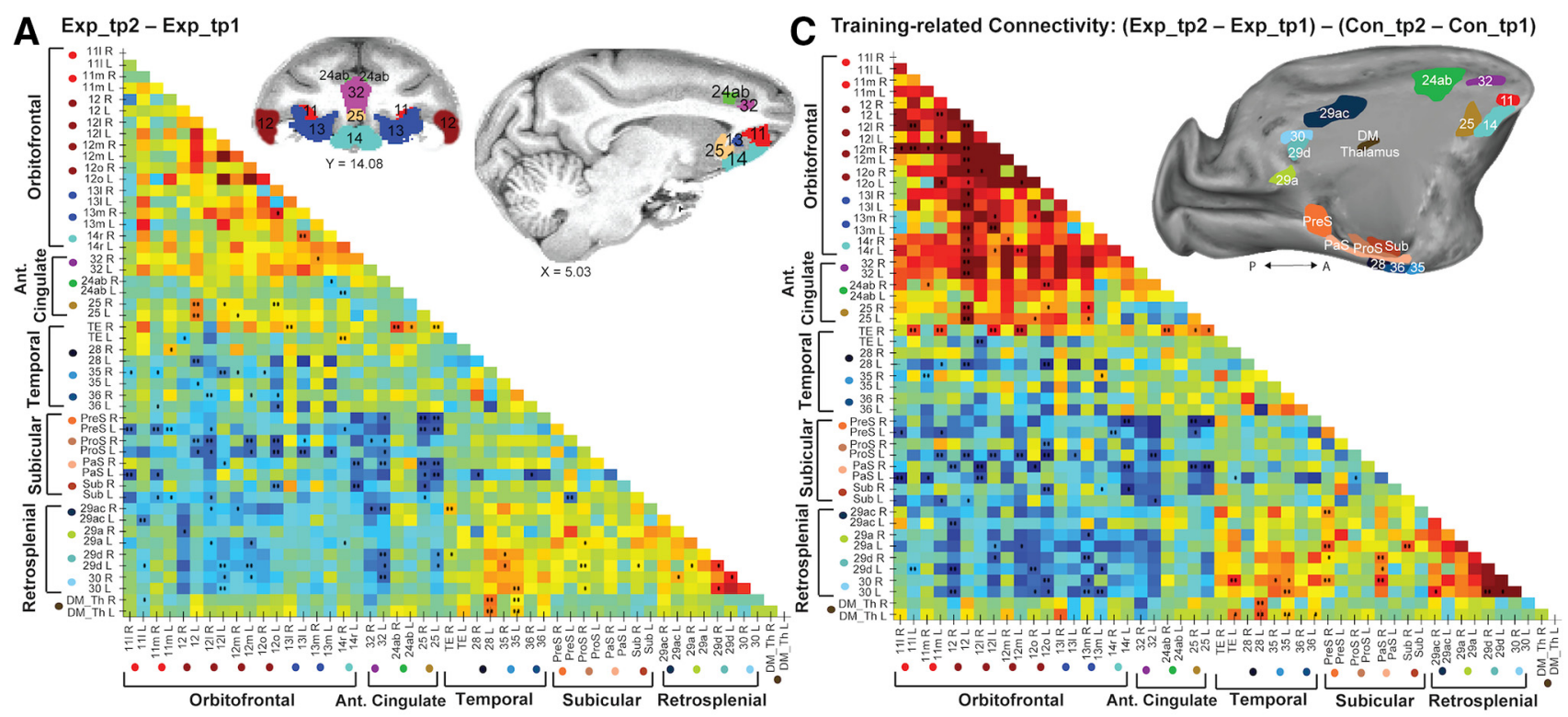

B Con_tp2-Con_tp1

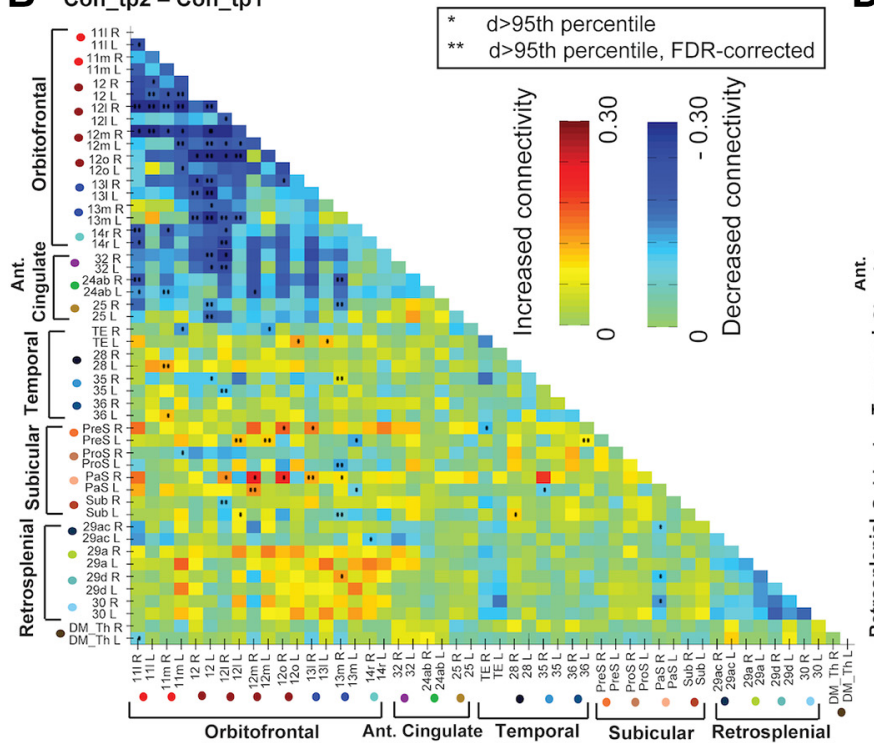

D Lesion-related Connectivity: Postop - Exp_tp2

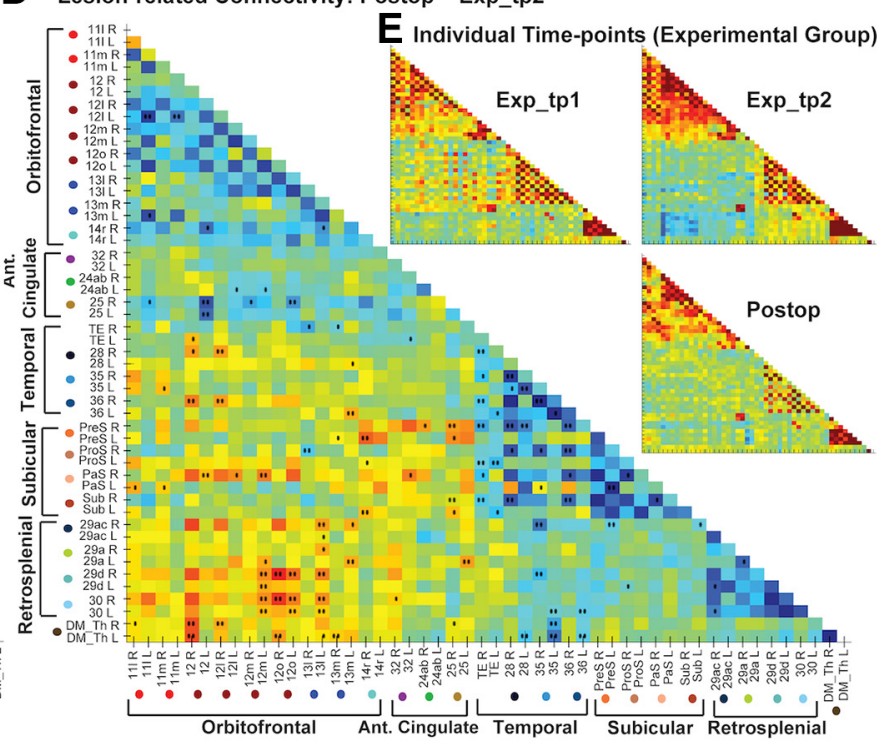

Figure 2. Connectivity matrices showing pairwise functional connectivity (defined as the rsfMRI time-series mean difference in correlations between pairs of ROls) changes within our ROI network of interest. ROls (except area TE) are depicted in volumetric (coronal and sagittal planes) and surface-based (sagittal plane, medial view) brain representations in the top row of the figure. Both brain representations were transformed to standard monkey space (MACAQUE-F99). The surface-based inflated hemisphere was generated using Caret5 (http://www.nitrc.org/ projects/caret/) (Van Essen et al., 2001). A, Anterior; P, posterior. For details on the selection of ROls and on our pipeline in the functional connectivity analysis, see rsfMRI ROls and Analysis of changes in functional connectivity. $\boldsymbol{A}, \boldsymbol{B}$, Changes in functional connectivity, that is, changes in correlation strength, between the two time points of the Experimental group $(\boldsymbol{A})$ and the Control group ( $\boldsymbol{B}$ ). Increased mean correlations (values $>0$ in the color map) indicate increased connectivity in time point 2 compared with time point 1 , whereas values $<0$ indicate decreased connectivity in time point 2. *Pairwise connections whose connectivity difference (d) was larger than the 95th percentile of a null distribution calculated after randomly shuffling all session labels. ${ }^{*} d>$ 95th percentile, after FDR correction for multiple comparisons (Benjamini and Hochberg, 1995). C, Visuospatial training-related changes in functional connectivity, that is, changes in the Experimental group (Exp_tp2-Exp_tp1) compared with the Control group (Con_tp2-Con_tp1). Both groups of monkeys participated in reward-based visual training tasks, but only the Experimental group completed the complex visuospatial discrimination learning task (see Materials and Methods). Values $>0$ indicate increased connectivity in the Experimental compared with the Control group, whereas values $<0$ indicate decreased connectivity in the Experimental group. $\boldsymbol{D}$, Changes in functional connectivity after completing the postoperative performance test (Postop; the time point for the Experimental group scans after bilateral fornix transections), relative to Exp_tp2 (the time point scans before completing the preoperative performance test). $\boldsymbol{C}, \boldsymbol{D}$, Statistical significance was assessed as described for $\boldsymbol{A}, \boldsymbol{B}$. $\boldsymbol{E}$, Functional connectivity in the three time points of the Experimental group (i.e., Exp_tp1; Exp_tp2; Postop) individually. $\mathrm{ROI}$ arrangement in these matrices is identical to the rest of the matrices in the figure.

humans (Lehman et al., 2011; Jbabdi et al., 2013; Zakszewski et al., 2014; Phillips et al., 2019). The fornix and UF have been strongly associated with learning and memory (see Introduction), and alterations of DTIderived microstructural measures have been reported in the VP of macaques following lesions of the hippocampus (Shamy et al., 2010; Meng et al., 2014).

We generated anatomic masks of our TOIs bilaterally, according to the macaque white matter atlas (Zakszewski et al., 2014). After TOIs were extracted from the atlas and masked, they were affine-transformed to the MACAQUE-F99 anatomic template. We intersected the masks with the mean FA skeleton mask obtained in TBSS (see Analysis of changes in structural connectivity), resulting in our final tract-specific masks (see Fig. 4).

\section{DTI statistics}

To identify which skeleton voxels underwent significant alterations because of our experimental manipulations, we performed statistical analyses on the FA maps using the "Randomise" algorithm that 
performs permutation-based nonparametric testing to control the familywise error (FWE) rate (Winkler et al., 2014). Significance $(p<0.05$, FWE-corrected) was established by running Randomise, in conjunction with the threshold-free cluster enhancement (TFCE) method (Smith and Nichols, 2009), which identifies clusters of difference in signal (in FA, in this case at a corrected significance level) without the need for specifying an arbitrary cluster-forming threshold. Randomise and TFCE are both part of the FSL suite. We used the default 5000 permutations in Randomise (although there is a limit in the number of possible permutations, determined by the number of subjects and design matrix in each experiment) (Winkler et al., 2014). Differences in the DTI-derived measures were calculated on a voxel-by-voxel basis in the skeletonized white matter TOIs. Statistical analyses were performed separately for each individual TOI bilaterally. The output images from Randomise were fed into FSL's "cluster" function used to find discrete clusters of significant ( $p=0.05$, FWE-corrected) voxels, and extract inferential statistics.

\section{Surgeries}

Detailed neurosurgical procedures for fornix transection have been previously published (Mitchell et al., 2008). Briefly, the surgeries were performed in a dedicated operating theater under aseptic conditions. Perioperative medication (as detailed in Buckley and Mitchell, 2016) was prescribed by veterinarians and administered by trained staff. For the fornix transection, a glass aspirator was used to make a sagittal incision no more than $10 \mathrm{~mm}$ in length in the corpus callosum at the level of the interventricular foramen. The fornix was sectioned transversely by electrocautery just above the interventricular foramen or anterior thalamus and aspirated with a 20 -gauge metal aspirator that was insulated to the tip. When the fornix transection was complete, the dura was repositioned and sewn, the bone flap was replaced and held with sutures, and the skin and galea were closed in layers.

After all neurosurgery, each monkey was monitored continuously for at least $48 \mathrm{~h}$. Postoperative medication continued in consultation with veterinary staff, as previously detailed (Buckley and Mitchell, 2016). Operated monkeys rejoined their social group as soon as practical after surgery, during the second postoperative recovery day. Postoperative recovery proceeded as expected with no adverse effects observed in any of the 4 monkeys.

\section{Histology}

After completion of all behavioral testing and scanning, each monkey with a fornix transection was sedated with ketamine $(10 \mathrm{mg} / \mathrm{kg})$, deeply anesthetized with intravenous barbiturate, and transcardially perfused with $0.9 \%$ saline followed by $10 \%$ formalin. The brains were cryoprotected in formalin sucrose and then sectioned coronally on a freezing microtome at $50 \mu \mathrm{m}$ thickness. A 1 -in-10 series of sections was collected throughout the cerebrum; these were mounted on gelatin-coated glass microscope slides and stained with cresyl violet.

\section{Assessment of the fornix lesions}

In three of the Experimental Group monkeys, the fornix was bilaterally transected in the region above the intraventricular foramen or the anterior thalamus (see Fig. 6). It was not possible to perform the fornix transection in exactly the same location in each monkey because of crossing arteries in the midline. In 1 monkey, the fornix was damaged only unilaterally. In all 4 monkeys, the corpus callosum had also been resectioned at the midline extending to $\sim 10 \mathrm{~mm}$ in length from above the intraventricular foramen to above the mediodorsal thalamus.

\section{Results}

\section{Preoperative behavioral performance}

During the course of the training sessions, the monkeys demonstrated that they improved in their ability to complete the OIP task (as measured by the mean percent errors per session and the mean time to complete each session across 10 consecutive training sessions). The mean percent errors made per session early in training (mean $=30.75, \mathrm{SD}=3.45)$, when the monkeys were completing their first 10 sessions of 10 trials per session repeated 8 times, were higher than mean errors made during completing 10 sessions late in training $($ mean $=13.86, \mathrm{SD}=4.80)$, on attaining the learning criterion before the preoperative test. A paired-sample two-tailed $t$ test confirmed that this difference in errors was significant $\left(t_{(3)}=10.00, p=0.002\right)$. Similarly, the mean time to complete each session early in training (mean $=28 \mathrm{~min}, \mathrm{SD}=3$ $\mathrm{min}$ ) was higher than late in training (mean $=23 \mathrm{~min}, \mathrm{SD}=6$ min). A paired-sample two-tailed $t$ test confirmed that this difference was significant $\left(t_{(3)}=3.83, p=0.031\right)$.

We also examined errors made by the monkeys within these same 10 training sessions early versus late in training, during different trial repetition runs within each session, namely, repetition runs 2 and 3, and repetition runs 7 and 8. Trial repetition runs 2 and 3 were the two repetitions of each of the trials immediately after the first presentation of the discriminations for each session; thus, at this time, these discriminations were still relatively novel. In contrast, trial repetition runs 7 and 8 were the repetition runs at the end of each session, when the monkeys had already been exposed to them a number of times within the session.

For repetition run 2, the mean percent errors made by the monkeys early in training (mean $=45, \mathrm{SD}=5.94$ ) were higher than late in training $($ mean $=29, \mathrm{SD}=4.90)$. A paired-sample two-tailed $t$ test showed that this difference was significant $\left(t_{(3)}=\right.$ $5.84, p=0.010$ ), indicating that the monkeys had learned how to learn the novel discriminations rapidly within one repetition, after many months of training on the task. Similarly, for repetition run 3 , the mean percent errors made by monkeys was higher early in training (mean $=38.5, \mathrm{SD}=4.20$ ) than late in training when the monkeys had mastered learning how to perform this task (mean $=23, \mathrm{SD}=6.22$ ). The paired-sample two-tailed $t$ test confirmed that this difference was significant $\left(t_{(3)}=5.05\right.$, $p=0.015$ ), although this result would not survive Bonferroni corrections for multiple $t$ test comparison (set at $p<0.0125$ ).

Further, for repetition run 7 , the mean percent errors made by the monkeys early in training (mean $=22.3, \mathrm{SD}=3.40$ ) was higher than that late in training (mean $=4.25, \mathrm{SD}=3.10$ ). A paired-sample two-tailed $t$ test showed that this difference was significant $\left(t_{(3)}=13.94, p=0.001\right)$, indicating further that the monkeys had learned how to learn the novel discriminations rapidly within each session after many months of training on the task. Similarly, for repetition 8 , the mean percent errors made by monkeys was higher early in training (mean $=20, S D=6.98$ ) than late in training, when the monkeys had mastered learning how to perform this task (mean $=4.5, \mathrm{SD}=2.38$ ). The pairedsample two-tailed $t$ test confirmed that this difference was significant $\left(t_{(3)}=6.46, p=0.008\right)$.

During the preoperative test, monkeys in the Experimental group continued to show good behavioral accuracy (Fig. 1C,D) with mean percent errors of $<15 \%$.

\section{Training-related changes in resting-state functional connectivity (rsfMRI)}

After the monkeys attained their consistent training criterion (Fig. 1C), we investigated changes in brain connectivity using rsfMRI. We collected longitudinal MRI data from our Experimental group, and our age- and cohort-matched Control group, both before (tp1) and after (tp2) training. Given our analyses involved multiple scans from the same monkeys over time, we performed control analyses to examine signal-to-noise ratio changes across time points. These two-tailed $t$ tests revealed no significant differences in signal-to-noise ratio within the Experimental group: Exp_tp1 versus Exp_tp2: $p=0.28$; Exp_tp1 versus Postop: $p=0.58$; Exp_tp2 versus Postop: $p=0.19$; within the Control group: Con_tp1 versus 
Con_tp2: $p=0.48$; or Between groups: Exp_tp1 versus Con_tp1: $p=0.47 ;$ Exp_tp2 versus Con_tp2: $p=0.53$.

The connectivity matrices in Figure 2 show how the changes (for each pair of ROIs, the mean difference between time points of rsfMRI time-series correlations) in resting-state functional connectivity changed over the two time points, and were, in general, driven by the Experimental group. After FDR correction, we identified connections for which the differences in functional connectivity at tp2 compared with tp1 were significantly different for the two contrasts: Experimental group, (Exp_tp2 - Exp_tp1); and Control group, (Con_tp2 - Con_tp1); and for the interaction, Experimental group versus Control group ([Exp_tp2 - Exp_tp1] [Con_tp2 - Con_tp1]).

Interestingly, in the functional connectivity matrix for the Experimental group (contrast: Exp_tp2 - Exp_tp1; Fig. 2A), significant functional connectivity changes between the two time points were observed in many of the brain areas involved in rapid associative learning of visuospatial discriminations. The smaller insets (Fig. 2E) showing the matrices for Exp_tp1 and Exp_tp2 highlight that high correlations of functional connectivity within the frontal cortex, within the retrosplenial cortex, within the subicular complex, and within the temporal lobes were generally maintained or increased between the two time points. Lateral inferotemporal cortex (TE) showed increased functional connectivity in tp2 compared with tp1 with OFC (areas 13 and 14). The DM_Th showed increased connectivity between entorhinal cortex (areas 28) and perirhinal cortex (area 35). Different parts of the retrosplenial cortex (RSC) showed increased connectivity with area $35, \mathrm{TE}$, and the ProS. While the functional connectivity for most parts of the frontal cortex remained unchanged, there were several significant increases between vmPFC (area 25) and ventrolateral PFC (area 12) and area 13; between area 14 and 13; and between ACC (area 24ab) and area 25 . In addition, there was a significant decrease in tp2 compared with tp 1 between area $24 \mathrm{ab}$ and area 14 . Functional connectivity also decreased between the temporal lobes in area 28, and areas 35 and 36 (perirhinal cortex), and area 12. The RSC also showed significant decreases with area 12 and dorsal ACC (area 32). Finally, significant decreases in functional connectivity were also observed between many parts of the subicular complex and the frontal cortex (areas 11, 12, 13, 25, and 32).

Intriguingly, for the Control group (contrast: Con_tp2 Con_tp1; Fig. 2B), the significant functional connectivity changes observed between the two time points were fewer and, in many cases, the reverse of those observed for the Experimental group. In particular, within the OFC (areas 11, 12, and 13), there were significant decreases in connectivity. Areas 14, 24ab, 25, and area 32 also showed significant decreases in connectivity with areas in the OFC. PreS and PaS showed increases with areas 12 and 13, while the ProS and subiculum (Sub) showed decreases with area 12. Finally, area 35 showed reduced connectivity with area 13 but increased connectivity with area 12, and area 28 showed increased connectivity with area 11.

Last, to determine whether there was an effect of training in the Experimental group compared with the Control group, an interaction matrix ([Exp_tp2 - Exp_tp1] - [Con_tp2 Con_tp1]) (Fig. 2C) was created. Notably, any significant interaction effects could reflect changes across time in the Experimental Group, the Control group, or both. Indeed, the data reveal that most of the functional connectivity changes between the two time points in this interaction are driven by changes in the Experimental group, or both the Experimental and Control groups. In particular, it is striking to see the significant decreases in functional connectivity in the Experimental group compared with the Control group, between different parts of the subicular complex or the retrosplenial cortex and the frontal cortex (areas 11, 12, 13, 14, 32, and 25). Areas 28, 35, and TE in the inferotemporal cortex also showed decreased connectivity with parts of areas 11 and 12. Further, this interaction matrix also highlights significant increases in functional connectivity in the Experimental group compared with the Control group within areas 11, 12, and 13; between area 12 and areas 14, 25, and 32; between TE and areas 11 and 12, and right area 24; within the RSC (areas 29 and 30); between the RSC and different parts of the subicular complex; and between the left hemisphere area 35, area 28, and the DM_Th (Fig. 2C).

Whole-brain seed-to-voxel connectivity maps (Fig. 3) using the right hemisphere 120 ROI as an example seed illustrate further changes with contrasts between the two time points for the Experimental group (Fig. $3 A$ ) and the Control group (Fig. 3B), and the training-related connectivity in the Experimental group compared with the Control group (Fig. 3D).

\section{Training-related changes in white matter connectivity}

We used DTI to evaluate structural connectivity and determine whether the functional connectivity changes we observed were accompanied by contiguous changes in the white matter anatomy. We explored white matter microstructural changes within the Experimental group by comparing their FA images at Exp_tp2 versus Exp_tp1 scans using a voxel-based approach (see DTI statistics) and paired-sample two-tailed $t$ tests, separately for each bilateral TOI. Strikingly, in addition to the rsfMRI changes within OFC, and to vmPFC and TE gray matter ROIs, our DTI analyses showed there was a significant white matter tract change within the Experimental Group in the UF, with significantly increased FA in Exp_tp2 relative to Exp_tp1 $(p=0.014)$ in a cluster of voxels in the right hemisphere (Table 1; Figs. $4 B, 5 A$ ). Similarly, in addition to the rsfMRI changes within the OFC and vmPFC gray matter ROIs, our DTI analyses showed there was a significant change within the Experimental Group for the VP with significantly decreased FA in Exp_tp2 compared with Exp_tp1 $(p=0.014)$ in a cluster of voxels in the left hemisphere (Table 1; Figs. 4C, 5C). Finally, FA significantly increased in Exp_tp2 relative to Exp_tp1 in one voxel in the fornix, located at the falx, after training ( $p=0.04$; Table 1; Figs. $4 D, 5 B)$.

In contrast, in our Control group of monkeys, FA measures between matched time points (Con_tp2 vs Con_tp1) showed no significant differences for any of the three TOIs (the voxel with the lowest $p$ value found across TOIs was $p=0.11$ ).

Finally, we ran a 2 (group) $\times 2$ (time point) interaction analysis ([Exp_tp2 - Exp_tp1] - [Con_tp2 - Con_tp1]). However, we observed no significant interaction using the TFCE correction. Nevertheless, given our rsfMRI findings, we explored the DTI data further using a more liberal, cluster-based, threshold of $z=1.6, p=0.05$ corrected. This revealed a cluster ( size $=96$ voxels) in right UF, covering some of the exact same voxels revealed in the Exp_tp2 - Exp_tp1 (TFCE) analysis (Fig. 4B).

For illustrative purposes, the percent and mean changes in FA within the UF and VP clusters and the single fornix voxel are shown in Figure 5 for individual monkeys in the Control and Experimental groups. As depicted, FA increased in the UF and in the fornix, and decreased in the VP, in both groups of monkeys (except in 1 Control monkey) over the two time points regardless of the visual task that they were learning (see Materials and Methods). Notably though, the FA change was 


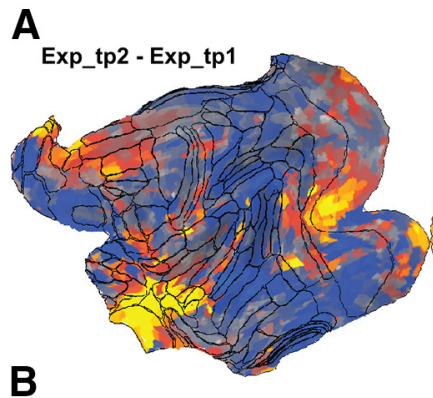

B Con_tp2 - Con_tp1
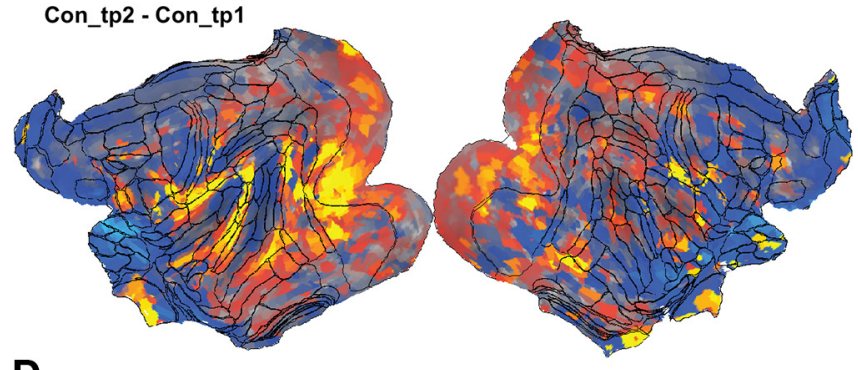

D

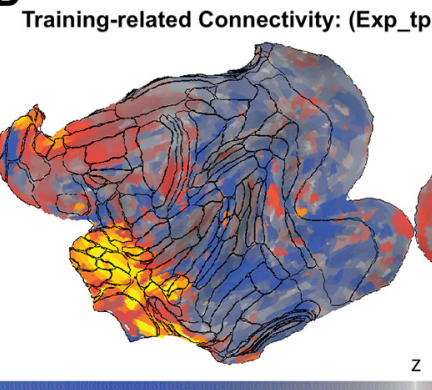

$-0.3$

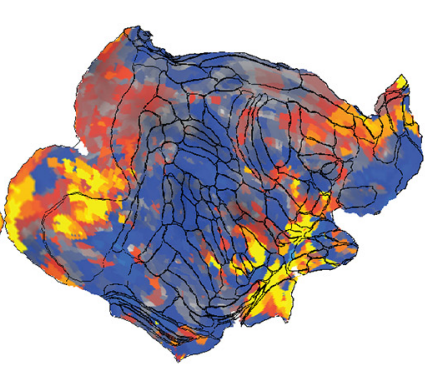

\begin{abstract}
tp2 - Exp_tp1) - (Con_tp2 - Con_tp1)
\end{abstract}

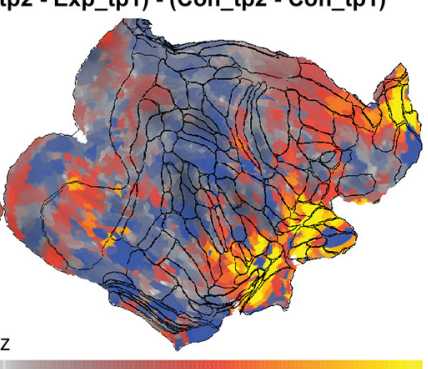

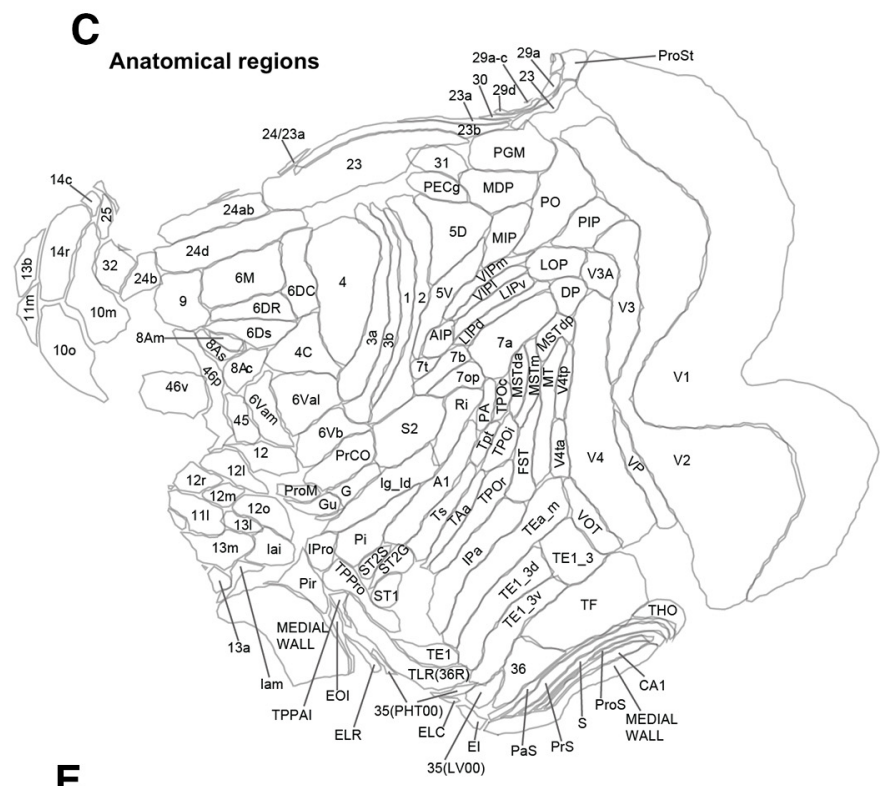

E

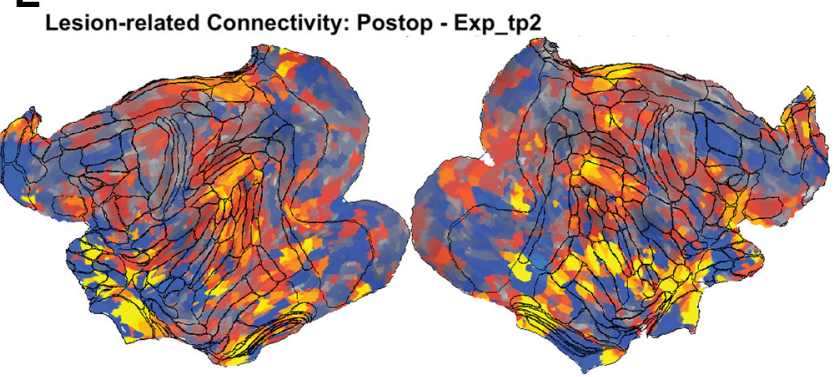

Figure 3. Whole-brain seed-to-voxel connectivity maps for the right hemisphere $120 \mathrm{ROI}$ as an example seed showing functional connectivity (defined as the rsfMRI time-series mean difference in correlations between our seed and all other voxels in the brain). Maps show changes in functional connectivity, that is, changes in correlation strength, between the two time points of the Experimental group $(\boldsymbol{A})$ and the Control group $(\boldsymbol{B})$. Increased mean correlations (values $>0$ in the color map) indicate increased connectivity in time point 2 compared with time point 1 , whereas values $<0$ indicate decreased connectivity in time point 2. C, Anatomical regions from the LV-FOA-PHT composite cytoarchitectonic parcellation (Van Essen et al., 2012). D, Visuospatial training-related changes in functional connectivity, that is, changes in the Experimental group (Exp_tp2-Exp_tp1) compared with the Control group (Con_tp2-Con_tp1). $E$, Changes in functional connectivity after completing the postoperative performance test (Postop; the time point for the Experimental group scans after bilateral fornix transections), relative to Exp_tp2 (the time point scans before completing the preoperative performance test).

Table 1. FA changes within the experimental group for changes after training was completed (Exp_tp2 - Exp_tp1) and for changes after bilateral fornix transection (Postop - Exp_tp2) ${ }^{\mathrm{a}}$

\begin{tabular}{|c|c|c|c|c|c|}
\hline \multirow[b]{2}{*}{ Cluster location } & \multirow[b]{2}{*}{ Direction of change } & \multirow[b]{2}{*}{ Hemisphere } & \multirow[b]{2}{*}{ Cluster size } & \multicolumn{2}{|l|}{ Peak voxel } \\
\hline & & & & Coordinates $(x, y, z)$ & $p$ \\
\hline \multicolumn{6}{|c|}{ Changes in FA after completing training in the visuospatial task (Exp_tp2 - Exp_tp1) } \\
\hline VP & FA decrease & Left & 119 & $-4.53,15.59,8.55$ & 0.014 \\
\hline Fornix & FA increase & Midline & 1 & $-0.00,1.01,6.04$ & 0.04 \\
\hline \multicolumn{6}{|c|}{ Lesion-related changes in FA after bilateral fornix transection (Postop - Exp_tp2) } \\
\hline
\end{tabular}

${ }^{a}$ The TFCE method (Smith and Nichols, 2009) was used to identify significant clusters. TFCE corrected for multiple comparisons with FWE rate under permutation testing for $\alpha=0.05$. Table shows the location and size (number of voxels) of the clusters found, and the MACAQUE-F99 coordinates (Van Essen, 2002) and TFCE-based $p$ value of the peak voxel in the cluster. Clusters were labeled based on a macaque white matter atlas (Zakszewski et al., 2014). Exp_tp2, the time point for the Experimental group scans after training was completed in the visuospatial task; Exp_tp1, the scans of the Experimental group before their training; Postop, the time point for the Experimental group scans after bilateral fornix transections.

much larger in all 4 monkeys in the Experimental Group at tp2 after completing training in the visuospatial task.

\section{Postoperative behavioral performance}

A subset of the monkeys in the Experimental group ( $n=3$ of 4 ) received bilateral fornix transection (Fig. 6). A single monkey in the Experimental group received unilateral damage to the fornix.

As Figure $1 C, D$ shows, after fornix transection, the monkeys with bilateral damage were cognitively impaired during rapid learning of new visuospatial discriminations within each testing session, as measured by an increase in the errors made as a function of trial repetition runs (Fig. 1D). A paired-sample $t$ test was used to compare the mean percent errors made during the preoperative test $($ mean $=13.76, \mathrm{SD}=1.78)$ with the mean percent errors made during the postoperative (bilateral damage) test (Bilat Fornix: mean $=35.43, \mathrm{SD}=5.22$ ). The $t$ test revealed a significant difference between the two tests $\left.t_{(2)}=5.45, p=0.032\right)$, indicating that, relative to their preoperative performance, the 
A
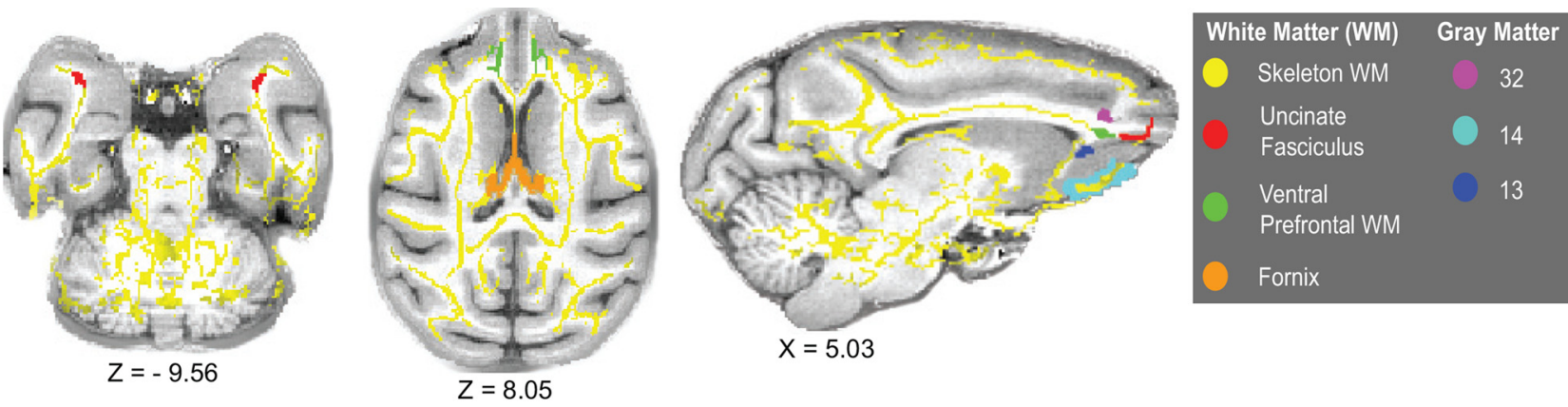

$X=5.03$
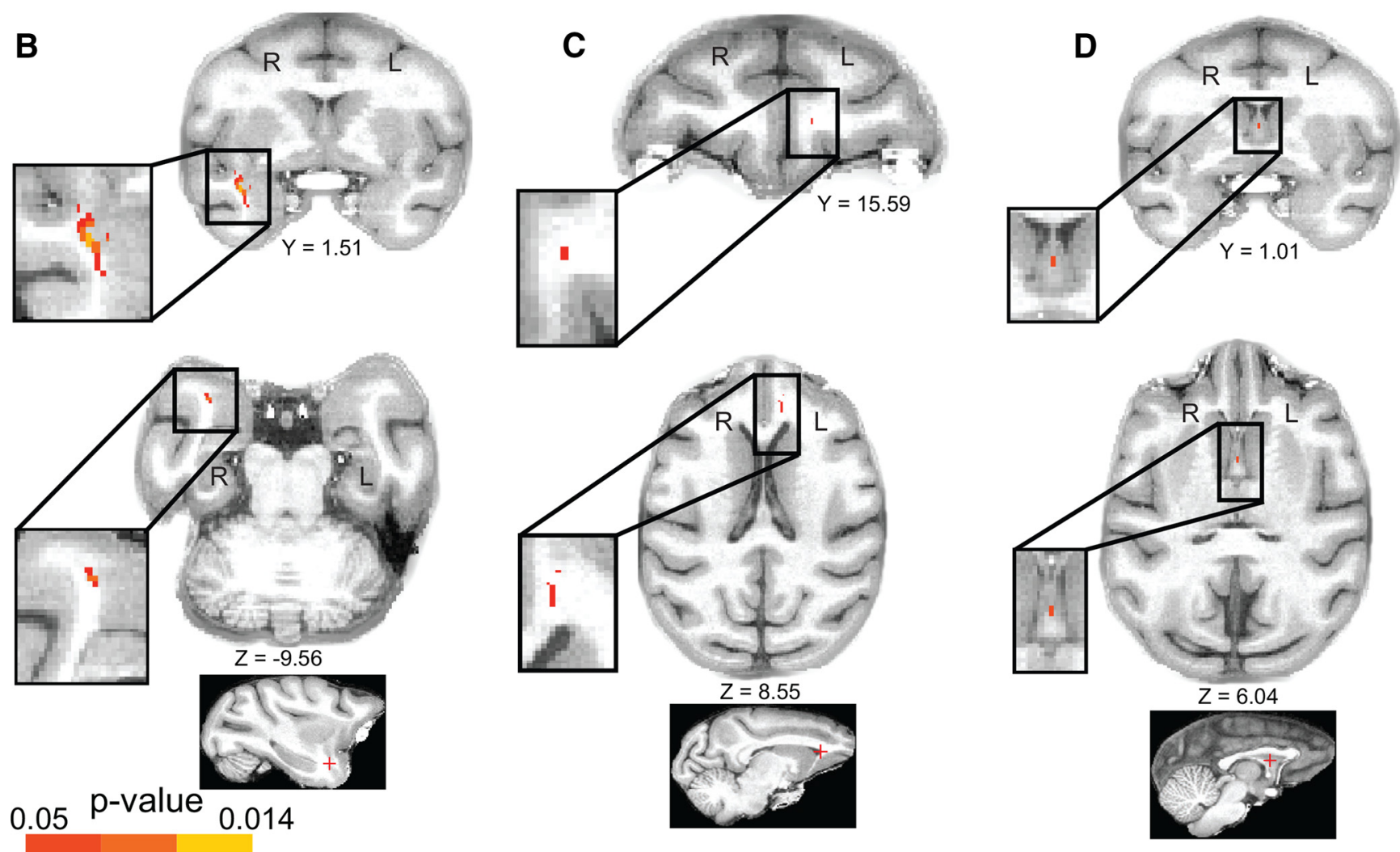

Figure 4. A, Transverse and sagittal views of the white matter TOls in our study and a selection of adjacent, key, prefrontal gray matter ROls for reference (for the entire set of ROls, see Fig. 2). Our TOls are as follows: the UF (red), VP white matter (green), and the fornix (orange). All TOl masks were intersected with the white matter skeleton FA mask (yellow; see Materials and Methods). All brain masks and the white matter skeleton were coregistered with the MACAQUE-F99 standard anatomic template (Van Essen, 2002). B-D, FA changes after training were completed in the visuospatial task. Coronal and transverse plane views of the $p$ value images coregistered with MACAQUE-F99. Colored voxels represent the clusters (threshold at $p<0.05$; TFCE-corrected for multiple comparisons) of significant FA differences within the Experimental group in tp2 relative to tp1. $B$, Significant FA increase in Exp_tp2 relative to Exp_tp1 in a cluster that corresponds to the UF (right hemisphere). C, Significant FA decrease in Exp_tp2 in a cluster that corresponds to the ventral prefrontal white matter (left hemisphere). D, Significant FA increase in Exp_tp2 in a single voxel in the fornix. As indicated by letters $R$ and $L$ superimposed on the figure, the left hemisphere is shown on the right of each image (radiologic display convention). Coordinates refer to MACAQUE-F99 space. The locations of the clusters (marked with a red cross) are also indicated in sagittal plane views, at the bottom, for reference.
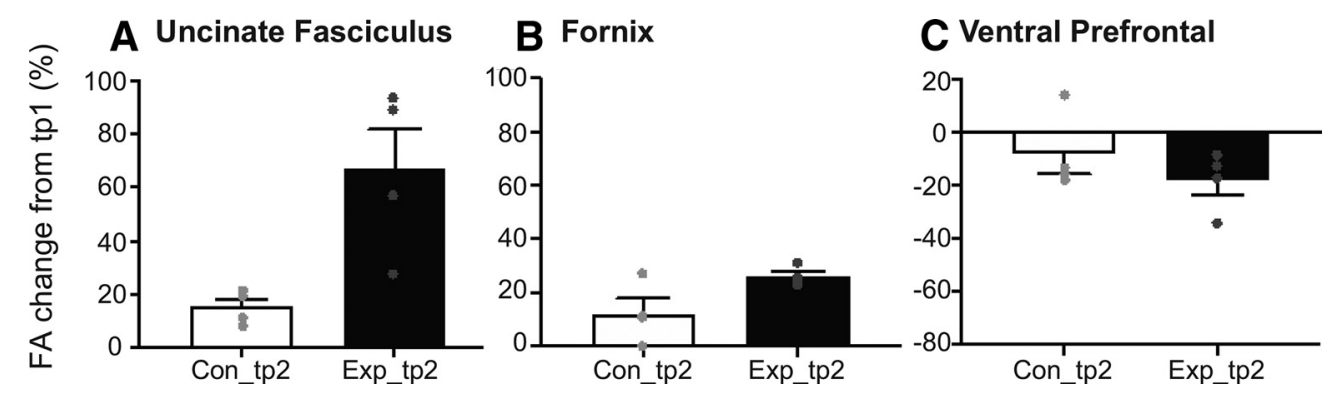

Figure 5. FA changes in the Experimental and Control Groups. Mean FA percent change from tp1 to tp2 for the Control (Con_tp2) and the Experimental (Exp_tp2) groups in (A) the uncinate fasciculus, $(\boldsymbol{B})$ the single voxel in the fornix, and $(\boldsymbol{C})$ the ventral prefrontal. Dots indicate FA change for individual monkeys. Error bars indicate SEM. 
monkeys with the bilateral fornix transection had a significant deficit in rapid learning of new visuospatial discriminations (Fig. 1D).

To assess how fornix damage impaired learning performance on the task, we ran similar analyses to those used to examine the effects of training on learning, by comparing performance during the preoperative test versus the postoperative test, with a particular focus on trial repetition runs 2 and 3, and trial repetition runs 7 and 8 . For repetition run 2 , the mean percent errors made by the monkeys during the preoperative test $($ mean $=34.33, \mathrm{SD}=1.53)$ was lower than the postoperative test (mean= $42.33, \mathrm{SD}=7.64$ ). However, a paired-sample two-tailed $t$ test confirmed that this difference was not significant $\left(t_{(2)}=1.98\right.$, $p=0.186$ ). Similarly, for repetition run 3 , the mean percent errors made by monkeys during the preoperative test $($ mean $=25.33, \mathrm{SD}=1.53$ ) was lower than the postoperative test (mean $=38.33, \mathrm{SD}=9.61$ ), with a paired-sample two-tailed $t$ test showing this difference was not significant $\left(t_{(2)}=2.53, p=0.127\right)$. For repetition run 7 , the mean percent errors made by the monkeys during the preoperative test (mean $=5.33, \mathrm{SD}=1.53$ ) was lower than during the postoperative test $($ mean $=26.67, \mathrm{SD}=7.64)$. A paired-sample two-tailed $t$ test revealed that this difference was significant $\left(t_{(2)}=4.354, p=\right.$ 0.049 ), although this result would not survive Bonferroni correction for multiple $t$ test comparisons (set at $p<0.0125$ ). Finally, for repetition run 8 , the mean percent errors made by monkeys were lower during the preoperative test (mean = $5.33, \mathrm{SD}=3.79$ ) than during the postoperative test (mean $=$ $24.67, \mathrm{SD}=7.23$ ), although again, the paired-sample two-tailed $t$ test confirmed that this difference was not significant $\left(t_{(2)}=3.05\right.$, $p=0.093)$.

We also compared the mean times to complete each session of the preoperative test (mean $=22.65, \mathrm{SD}=6.24)$ and the postoperative test $($ mean $=29.30, S D=1.10)$. A paired-sample twotailed $t$ test showed there was no significant difference $\left(t_{(2)}=\right.$ 1.28, $p=0.329)$. Thus, while the fornix transection caused the monkeys to make more errors in their performance, it did not significantly affect their ability to complete the task, suggesting a specific lesion-related deficit in rapid associative learning for new visuospatial discriminations, rather than an inability to perform the task (i.e., their procedural memory remained intact). This specific deficit in new learning accords with a study in humans (Tsivilis et al., 2008) that found that 3 patients with bilateral fornix damage had substantially lower long-term memory performance than expected based on the patients' intellectual and intelligence scores (yet their working memory performance was unimpaired).

The 1 monkey with the unilateral fornix damage continued to show intact learning of new visuospatial discriminations (Fig. 1C,D). These observations further support the notion that the neurosurgical procedures, the perioperative drug treatments, or the surgical approach of slicing through up to 10 $\mathrm{mm}$ of corpus callosum at the midline (but not transecting it) to expose and transect the fornix fibers underneath did not in themselves result in the cognitive deficits observed in the bilateral lesion group or in the brain connectivity changes (described below). Instead, the lack of change in cognitive ability specific to this task in the unilaterally damaged monkey is consistent with findings in humans who have undergone neurosurgery to remove a colloid cyst growing in the fornix or around the third ventricle; a procedure that causes damage to the fornix but typically not a complete transection of the fibers (Aggleton et al., 2000).

\section{Lesion-related changes in resting-state functional connectivity}

Intriguingly, the consequence of the fornix transection in our well-trained Experimental group monkeys had a different, but not necessarily opposing, effect on the functional connectivity profile compared with the impact of preoperative training (Fig. 2). Notably, the increased connectivity profile within the PFC, within the subicular complex, within the temporal lobes, and within the RSC after training (Fig. 2E) was reduced in the postop scan (with the exception of area 25), while the training-induced connectivity changes between PFC and other areas, and between the DM_Th and temporal areas, were generally reversed (Fig. 2D). In addition, decreased connectivity was observed among temporal and subicular areas, which had not been significantly affected by training (Fig. 2D). Whole-brain seed-to-voxel connectivity maps using the right hemisphere 12o ROI as an example seed illustrate further changes for the lesion-related connectivity in the Experimental group (Fig. 3E). Thus, consistent with results from previous lesion studies, the fornix transection caused widespread disruption to many, distributed neural networks (Gaffan, 2002; Mitchell et al., 2008). We used the same analysis pipeline to compare changes in the mean correlations between pairs of ROIs in the scans of the Experimental group after bilateral fornix transection (Postop) with the mean correlations acquired in their scans after mastering the task (Exp_tp2). As Figure 2D shows, bilateral fornix transection caused increased functional connectivity between the DM_Th and areas 12 and 13 and area 25; between the RSC and areas 12 and 13; between the PaS and area 12; between the PreS and area 25; and between areas 28 and 36 and areas 12 and 13. We also observed decreased functional connectivity between area 25 and area 12; between TE, and areas 28, 36, PreS, ProS, and Sub; between areas 35 and 36, and the areas 29 and 30, and the DM_Th, and PreS, ProS, and Sub; and within the subicular complex itself. 

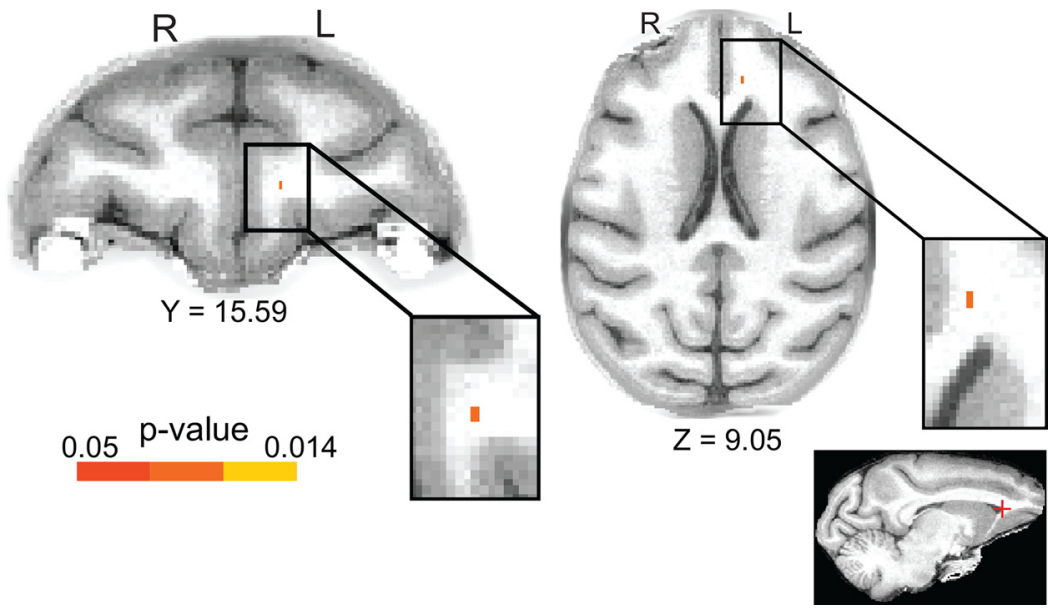

Figure 7. Coronal and transverse plane views of the $p$ value images coregistered with the standard anatomic template (MACAQUE-F99) for reference. Colored voxels represent the cluster (threshold at $p<0.05$; TFCE-corrected for multiple comparisons) of significant FA increases in Postop (the time point for the Experimental group scans after the bilateral fornix transection and completing the postoperative performance test) and Exp_tp2 (the time point for the Experimental group scans after completing training and their preoperative performance test). Cluster corresponds to the ventral prefrontal white matter (left hemisphere). $R$ and $L$ indicate the hemisphere. Coordinates refer to MACAQUE-F99 space. The location of the cluster (marked with a red cross) is also indicated in a sagittal plane view, at the bottom of the figure, for reference.

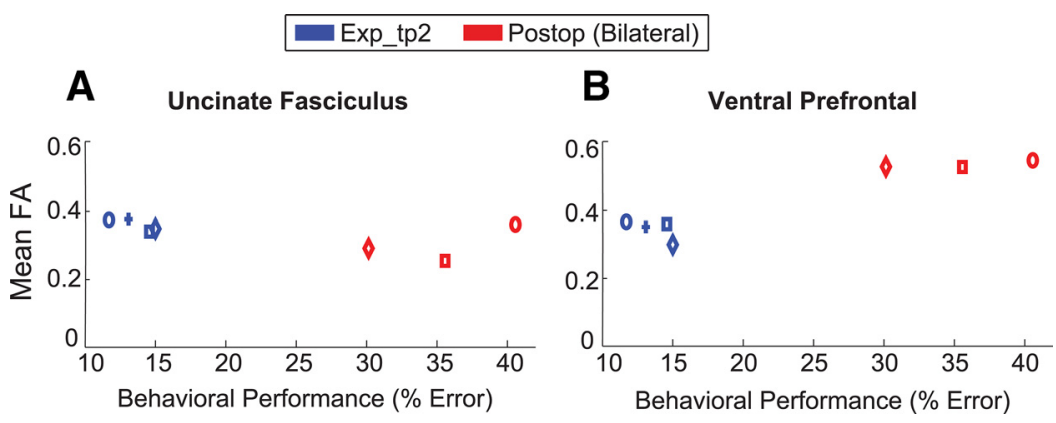

Figure 8. Mean FA as a function of preoperative (Exp_tp2) and postoperative (Postop) behavioral performances in the monkeys trained on the visuospatial task. FA was measured in the uncinate fasciculus $(\boldsymbol{A})$ and the ventral prefrontal $(\boldsymbol{B})$ clusters, identified from the Exp_tp2 versus Exp_tp1, and the Postop versus Exp_tp2 contrasts, respectively. FA differences between Exp_tp2 and Postop were not significant within the UF cluster but were significant within the ventral prefrontal cluster (the cluster is defined on this basis). Each marker represents the individual monkey's mean performance, and the same symbol across colors signifies the same monkey. Blue cross symbol represents the unilateral fornix lesion monkey; hence, it is not represented in the Postop bilateral panel. The preoperative and postoperative behavioral tests took place around the monkeys' Exp_tp2 and Postop scans, respectively; therefore, the terminology used in the figure legend here refers to both the behavioral and MRI data.

Lesion-related changes in white matter connectivity

To evaluate the white matter changes associated with bilateral fornix transection in our trained monkeys, we compared FA between Postop and Exp_tp2. Given that the fornix was transected in the Postop scans, we did not include any fornix masks in these analyses. Remarkably, in addition to the changes in OFC and vmPFC functional connectivity with the DM_Th, our DTI analyses showed that after bilateral fornix transection, FA increased significantly in the Postop relative to Exp_tp2 $(p=0.029)$ in the left hemisphere VP (Table 1; Figs. 7, 8B). Notably though, FA measures in the training-related right UF cluster remained unchanged, which is similar to a previous study that has reported no changes to UF after hippocampal lesions in humans (Henson et al., 2016). Figure 8 shows descriptively, the mean FA in VP and UF linked to each monkey's performance in the visuospatial task, before and after bilateral fornix transection.

\section{Discussion}

Using a complex visuospatial cognitive task and longitudinal study design, we captured learning-related corticocortical and thalamocortical structural and functional connectivity changes. To our knowledge, this study details, for the first time, these connectivity changes in the primate brain, linked to learning-to-learn novel visuospatial discriminations. After learning, our Experimental monkeys showed functional connectivity changes between the OFC, vmPFC, and inferotemporal cortex and within the subicular complex, and between parts of the subicular complex, and RSC, and frontal cortex. Structural changes to the conjunct UF, fornix, and VP white matter tracts were also observed. Our study also uniquely shows that thalamocortical functional connectivity from the inferotemporal and entorhinal cortex to the DM_Th was increased after learning, while the VP white matter tract had reduced FA. Connectivity changes in the Experimental group were larger than in an age- and cohort-matched control group, who had learned an unrelated visual task (Pelekanos et al., 2020). In accord with our findings, strong links between functional and structural connectivity have been reported in the healthy brain (Honey et al., 2007, 2009; O'Reilly et al., 2013).

To our knowledge, we also report previously unknown brain changes after fornix transection. As predicted, the monkeys were impaired in new learning. Interestingly, the increased functional connectivity observed within frontal, RSC, and temporal areas after learning was preserved, although reduced in magnitude (Fig. 2E). In contrast, functional connectivity was altered, but not always opposing, between interconnected areas. Specifically, functional connectivity increased between the RSC and frontal cortex, and between the DM_Th and frontal cortex, while it decreased between inferotemporal cortex, the subicular complex, and the DM_Th. The alterations to thalamocortical connectivity suggest changes in the structural architecture of the frontal network (linked to meta-reinforcement learning proposals) (Wang et al., 2018) was contributing to the learning deficits observed. There were also dissociable changes to FA, with an increase in the VP tract, but no structural connectivity changes in the UF. These changes between ventral PFC structures suggest the flexible use of rules needed to solve the task were disrupted (Wilson et al., 2014; Behrens et al., 2018).

After training, functional connectivity within and between many gray matter regions (including the extended hippocampal system, cingulate cortex, and ventral PFC) changed for the Experimental group. We observed increased connectivity within the OFC and RSC, and between OFC and vmPFC, and TE, between RSC and the subicular complex, and between entorhinal and perirhinal cortex and DM_Th. Our results are consistent 
with previous findings in humans that indicate increased activation in the subgenual vmPFC with memory consolidation linked to rewards (Takashima et al., 2006, 2007; Gais et al., 2007; Sterpenich et al., 2009) and in the temporal cortex after pictorial paired associate learning (Yamashita et al., 2009).

Our rsfMRI findings also accord with monkey lesion studies that selectively manipulated these frontal structures and DM_Th to assess learning new visuospatial discriminations (Parker and Gaffan, 1997; Baxter et al., 2007; Mitchell et al., 2007; Mitchell and Gaffan, 2008; Browning et al., 2015; Chakraborty et al., 2019). Lateral OFC and its underlying white matter tracts also contribute to reward guided learning in macaques (Noonan et al., 2010; Rudebeck et al., 2017), while in humans, subgenual vmPFC and OFC damage disrupts optimal learning and decision-making performance (Petrides, 1985; Bechara et al., 2000; Bright et al., 2006).

Interestingly, manipulations to primate cingulate, RSC, or entorhinal cortex significantly disrupted the recall and retention of previously learned visuospatial discriminations but left new learning intact (Parker and Gaffan, 1997; Mitchell et al., 2008; Buckley and Mitchell, 2016). Here, high positive functional connectivity correlations in the cingulate and RSC were observed across all three scans for the Experimental group (Fig. 2E), suggesting these regions do not underpin new learning but rather contribute to other cognitive processes that subserve visuospatial memory. This suggestion is supported by electrophysiological recordings from macaque cingulate cortex showing that this region contributes to monitoring the reward associated with learned actions selected, rather than computing these actions themselves ( $\mathrm{Li}$ et al., 2019). Moreover, while the functional contributions of the RSC are still to be determined, current evidence highlights its involvement in maintaining memories (Mitchell et al., 2018). White matter structural connectivity was also altered after training. As predicted, our DTI results showed that FA increased in the right hemisphere UF, and in the fornix, while FA decreased in a cluster of voxels in the left VP. Importantly, the UF and the VP tracts connect many of the conjunct gray matter structures in which we observed functional connectivity changes.

In primates, complex visual representations of objects and features are processed (though not exclusively) through the ventral visual processing stream in the inferotemporal cortex (Ettlinger, 1959; Ungerleider and Mishkin, 1982) before being transferred into the ventral PFC, via the UF (Ungerleider et al., 1989). Further, cross-sectional neuroimaging studies in healthy humans show UF FA changes correlated with episodic memory task performance and visual association learning (MetzlerBaddeley et al., 2011; Charlton et al., 2013; Thomas et al., 2015; Alm et al., 2016).

To our knowledge, the training-related FA decrease in the right VP is unique to our study. The VP contributes to the white matter connectivity among the ventral PFC (Lehman et al., 2011). We know that gray and white matter in the ventral PFC of macaques differentially contributes to visuospatial discrimination learning, probabilistically rewarded object learning and updating, reversal learning, and reward devaluation tasks (Izquierdo et al., 2004; Baxter et al., 2007; Chau et al., 2015; Rudebeck et al., 2017). It may be that the reduced FA measured in VP after mastering our visuospatial task is a consequence of sharpened ventral PFC response dynamics to the task demands, although further studies will need to investigate this proposal.

Finally, we observed significant FA change in the fornix, but only in one voxel, raising the question of why more extensive changes were not found. Notably, the human fornix is difficult to measure consistently as its thickness and fiber densities vary across the tract (Rudebeck et al., 2009). Further, while fornix damage impairs new visuospatial learning in monkeys and humans (Aggleton et al., 2000; Gaffan, 2002), and fornix white matter integrity correlates with cognitive functioning (Douaud et al., 2013), neuroimaging studies in healthy humans suggest only specific parts of the fornix support the recall and recollection of scenes (Rudebeck et al., 2009; Metzler-Baddeley et al., 2012) and scene discriminations (Tsivilis et al., 2008; Hodgetts et al., 2015). Thus, in the dramatically smaller monkey brain, any further FA microstructural changes may have been too slight to detect. We should also note that the FA changes we found were overall small across white matter tracts. This is possibly related to the statistical approach (TFCE FWE-corrected) we used to identify regions of significant FA change in any given test. More generally, it is also possible that the diffusion-tensor model we used is suboptimal for detecting small changes in the structure of fiber tracts with complex anatomy.

Bilateral fornix damage, as predicted, significantly impaired rapid learning of new visuospatial discriminations in the monkeys. In addition, we observed increased functional connectivity between the RSC and DM_Th and area 12, and decreased functional connectivity between the OFC and vmPFC, and within the inferotemporal cortex. Also, FA increased in a cluster of voxels in the VP white matter, compared with the monkeys' own presurgical measures of FA, while FA remained unchanged in the UF.

In other macaque neuroimaging studies, similar increases in DTI-derived white matter structural measures were reported in the VP following hippocampal lesions (Shamy et al., 2010; Meng et al., 2014). The FA increase in the VP also contrasts well with our finding of decreased VP FA on attaining stable task performance after training, before neurosurgery. The lack of FA change in the UF also accords with our otherwise healthy monkeys showing new learning deficits but demonstrating intact procedural memory for the task requirements. Interestingly though, reduced microstructural integrity of the UF has been correlated with diminished episodic memories in healthy elderly with a genetic risk of developing Alzheimer's disease (Lancaster et al., 2016), and in people with mild cognitive impairment, as well as other neuropsychiatric disorders and cognitive deficits (Fujie et al., 2008; Von Der Heide et al., 2013; Zhuang et al., 2013; Christidi et al., 2014; Hiyoshi-Taniguchi et al., 2015; Wang et al., 2017). Consequently, these contrasting results suggest reduced FA changes in the UF are potentially linked to changes in procedural memory performance in patient groups with cognitive impairments. Intriguingly, UF transection in monkeys abolishes the previously acquired learning-to-learn ability observed in discrimination learning set paradigms (Browning and Gaffan, 2008).

In conclusion, our current findings highlight that different, interdependent corticocortical and thalamocortical neural networks interact about how to learn, and optimize rapid new associative learning of visuospatial discriminations. Understanding how these networks function together in the normal primate brain has the potential for earlier detection of brain changes in neurodegenerative disorders that affect reward-guided learning and memoryguided decision-making abilities, and identify specific targets for more effective treatment options in the disrupted brain.

\section{References}

Aggleton JP, Brown MW (2006) Interleaving brain systems for episodic and recognition memory. Trends Cogn Sci 10:455-463. 
Aggleton JP, Desimone R, Mishkin M (1986) The origin, course, and termination of the hippocampothalamic projections in the macaque. J Comp Neurol 243:409-421.

Aggleton JP, McMackin D, Carpenter K, Hornak J, Kapur N, Halpin S, Wiles CM, Kamel H, Brennan P, Carton S, Gaffan D (2000) Differential cognitive effects of colloid cysts in the third ventricle that spare or compromise the fornix. Brain 123:800-815.

Aggleton JP, Vann SD, Saunders RC (2005) Projections from the hippocampal region to the mammillary bodies in macaque monkeys. Eur J Neurosci 22:2519-2530.

Aggleton JP, Wright NF, Rosene DL, Saunders RC (2015) Complementary patterns of direct amygdala and hippocampal projections to the macaque prefrontal cortex. Cereb Cortex 25:4351-4373.

Aggleton JP, Pralus A, Nelson AJ, Hornberger M (2016) Thalamic pathology and memory loss in early Alzheimer's disease: moving the focus from the medial temporal lobe to Papez circuit. Brain 139:1877-1890.

Ainsworth M, Browncross H, Mitchell DJ, Mitchell AS, Passingham RE, Buckley MJ, Duncan J, Bell AH (2018) Functional reorganisation and recovery following cortical lesions: a preliminary study in macaque monkeys. Neuropsychologia 119:382-391.

Alm KH, Rolheiser T, Olson IR (2016) Inter-individual variation in frontotemporal connectivity predicts the ability to learn different types of associations. Neuroimage 132:213-224.

Andersson JL, Skare S, Ashburner J (2003) How to correct susceptibility distortions in spin-echo echo-planar images: application to diffusion tensor imaging. Neuroimage 20:870-888.

Baxter MG, Gaffan D, Kyriazis DA, Mitchell AS (2007) Orbital prefrontal cortex is required for object-in-place scene memory but not performance of a strategy implementation task. J Neurosci 27:11327-11333.

Beaulieu C, Donahue CJ, Glasser MF, Preuss TM, Rilling JK, Van Essen DC (2002) The basis of anisotropic water diffusion in the nervous system: a technical review. NMR Biomed 15:435-455.

Bechara A, Tranel D, Damasio H (2000) Characterization of the decisionmaking deficit of patients with ventromedial prefrontal cortex lesions. Brain 123:2189-2202.

Behrens TE, Muller TH, Whittington JC, Mark S, Baram AB, Stachenfeld KL, Kurth-Nelson Z (2018) What is a cognitive map? Organizing knowledge for flexible behavior. Neuron 100:490-509.

Behzadi Y, Restom K, Liau J, Liu TT (2007) A component based noise correction method (CompCor) for BOLD and perfusion based fMRI. Neuroimage 37:90-101.

Benjamini Y, Hochberg Y (1995) Controlling the false discovery rate: a practical and powerful approach to multiple testing. J R Stat Soc B 57:289300 .

Bocchetta M, Iglesias JE, Neason M, Cash DM, Warren JD, Rohrer JD (2020) Thalamic nuclei in frontotemporal dementia: mediodorsal nucleus involvement is universal but pulvinar atrophy is unique to C9orf72. Hum Brain Mapp 41:1006-1016.

Braak H, Braak E (1991) Alzheimer's disease affects limbic nuclei of the thalamus. Acta Neuropathol 81:261-268.

Bright B, Buckman J, Fradera A, Yoshimasu H, Colchester AC, Kopelman MD (2006) Retrograde amnesia in patients with hippocampal, medial temporal, temporal lobe, or frontal pathology. Learn Mem 13:545-557.

Browning PG, Gaffan D (2008) Impairment in object-in-place scene learning after uncinate fascicle section in macaque monkeys. Behav Neurosci 122:477-482.

Browning PG, Chakraborty S, Mitchell AS (2015) Evidence for mediodorsal thalamus and prefrontal cortex interactions during cognition in macaques. Cereb Cortex 25:4519-4534.

Buckley MJ, Gaffan D (1998) Perirhinal cortex ablation impairs configural learning and paired-associate learning equally. Neuropsychologia 36: 535-546.

Buckley MJ, Mitchell AS (2016) Retrosplenial cortical contributions to anterograde and retrograde memory in the monkey. Cereb Cortex 26:29052918.

Chakraborty S, Ouhaz Z, Mason S, Mitchell AS (2019) Macaque parvocellular mediodorsal thalamus: dissociable contributions to learning and adaptive decision-making. J Neurosci 49:1041-1054.

Charlton RA, Barrick TR, Markus HS, Morris RG (2013) Verbal working and long-term episodic memory associations with white matter microstructure in normal aging investigated using tract-based spatial statistics. Psychol Aging 28:768-777.
Chau BK, Sallet J, Papageorgiou GK, Noonan MP, Bell AH, Walton ME, Rushworth MF (2015) Contrasting roles for orbitofrontal cortex and amygdala in credit assignment and learning in macaques. Neuron 87:1106-1118.

Christidi F, Zalonis I, Kyriazi S, Rentzos M, Karavasilis E, Wilde EA, Evdokimidis I (2014) Uncinate fasciculus microstructure and verbal episodic memory in amyotrophic lateral sclerosis: a diffusion tensor imaging and neuropsychological study. Brain Imaging Behav 8:497-505.

D’Esposito M, Verfaellie M, Alexander MP, Katz DI (1995) Amnesia following traumatic bilateral fornix transection. Neurology 45:1546-1550.

Douaud G, Menke RA, Gass A, Monsch AU, Rao A, Whitcher B, Zamboni G, Matthews PM, Sollberger M, Smith S (2013) Brain microstructure reveals early abnormalities more than two years prior to clinical progression from mild cognitive impairment to Alzheimer's disease. J Neurosci 33:2147-2155.

Eichenbaum H (2017) Prefrontal-hippocampal interactions in episodic memory. Nat Rev Neurosci 18:547-558.

Ettlinger G (1959) Visual discrimination following successive temporal ablations in monkeys. Brain 82:232-250.

Fahy FL, Riches IP, Brown MW (1993) Neuronal activity related to visual recognition memory: long-term memory and the encoding of recency and familiarity information in the primate anterior and medial inferior temporal and rhinal cortex. Exp Brain Res 96:457-472.

Fujie S, Namiki C, Nishi H, Yamada M, Miyata J, Sakata D, Sawamoto N, Fukuyama H, Hayashi T, Murai T (2008) The role of the uncinate fasciculus in memory and emotional recognition in amnestic mild cognitive impairment. Dement Geriatr Cogn Disord 26:432-439.

Gaffan D (1994) Scene-specific memory for objects: a model of episodic memory impairment in monkeys with fornix transection. J Cogn Neurosci 6:305-320.

Gaffan D (2002) Against memory systems. Philos Trans R Soc Lond B Biol Sci 357:1111-1121.

Gais S, Albouy G, Boly M, Dang-Vu TT, Darsaud A, Desseilles M, Rauchs G, Schabus M, Sterpenich V, Vandewalle G, Maquet P, Peigneux P (2007) Sleep transforms the cerebral trace of declarative memories. Proc Natl Acad Sci USA 104:18778-18783.

Genovese CR, Lazar NA, Nichols T (2002) Thresholding of statistical maps in functional neuroimaging using the false discovery rate. Neuroimage 15:870-878.

Henson RN, Greve A, Cooper E, Gregori M, Simons JS, Geerligs L, Erzinçlioğlu S, Kapur N, Browne G (2016) The effects of hippocampal lesions on MRI measures of structural and functional connectivity. Hippocampus 26:1447-1463.

Hiyoshi-Taniguchi K, Oishi N, Namiki C, Miyata J, Murai T, Cichocki A, Fukuyama $\mathrm{H}$ (2015) The uncinate fasciculus as a predictor of conversion from amnestic mild cognitive impairment to Alzheimer disease. J Neuroimaging 25:748-753.

Hodgetts CJ, Postans M, Shine JP, Jones DK, Lawrence AD, Graham KS (2015) Dissociable roles of the inferior longitudinal fasciculus and fornix in face and place perception. Elife 4:e07902.

Honey CJ, Kotter R, Breakspear M, Sporns O (2007) Network structure of cerebral cortex shapes functional connectivity on multiple time scales. Proc Natl Acad Sci USA 104:10240-10245.

Honey CJ, Sporns O, Cammoun L, Gigandet X, Thiran JP, Meuli R, Hagmann P (2009) Predicting human resting-state functional connectivity from structural connectivity. Proc Natl Acad Sci USA 106:2035-2040.

Huang AS, Rogers BP, Woodward ND (2019) Disrupted modulation of thalamus activation and thalamocortical connectivity during dual task performance in schizophrenia. Schizophr Res 210:270-277.

Hutchison RM, Hutchison M, Manning KY, Menon RS, Everling S (2014) Isoflurane induces dose-dependent alterations in the cortical connectivity profiles and dynamic properties of the brain's functional architecture. Hum Brain Mapp 35:5754-5775.

Insausti R, Amaral DG (2008) Entorhinal cortex of the monkey: IV. Topographical and laminar organization of cortical afferents. J Comp Neurol 509:608-641.

Izquierdo A, Suda RK, Murray EA (2004) Bilateral orbital prefrontal cortex lesions in rhesus monkeys disrupt choices guided by both reward value and reward contingency. J Neurosci 24:7540-7548.

Jbabdi S, Lehman JF, Haber SN, Behrens TE (2013) Human and monkey ventral prefrontal fibers use the same organizational principles to reach their targets: tracing versus tractography. J Neurosci 33:3190-3201. 
Jenkinson M (1999) Measuring transformation error by RMS deviation. Internal Technical Report TR99MJ1. Oxford: FMRIB Centre, University of Oxford. Available at https://www.fmrib.ox.ac.uk/analysis/techrep.

Krayniak PF, Siegel A, Meibach RC, Fruchtman D, Scrimenti M (1979) Origin of the fornix system in the squirrel monkey. Brain Res 160:401411.

Kwok SC, Mitchell AS, Buckley MJ (2015) Adaptability to changes in temporal structure is fornix-dependent. Learn Mem 22:354-359.

Lancaster MA, Seidenberg M, Smith JC, Nielson KA, Woodard JL, Durgerian S, Rao SM (2016) Diffusion tensor imaging predictors of episodic memory decline in healthy elders at genetic risk for Alzheimer's disease. J Int Neuropsychol Soc 22:1005-1015.

Le Bihan D (2003) Looking into the functional architecture of the brain with diffusion MRI. Nat Rev Neurosci 4:469-480.

Lee AC, Buckley MJ, Pegman SJ, Spiers H, Scahill VL, Gaffan D, Bussey TJ, Davies RR, Kapur N, Hodges JR, Graham KS (2005) Specialization in the medial temporal lobe for processing of objects and scenes. Hippocampus 15:782-797.

Lehman JF, Greenberg BD, McIntyre CC, Rasmussen SA, Haber SN (2011) Rules ventral prefrontal cortical axons use to reach their targets: implications for diffusion tensor imaging tractography and deep brain stimulation for psychiatric illness. J Neurosci 31:10392-10402.

Li YS, Nassar MR, Kable JW, Gold JI (2019) Individual neurons in the cingulate cortex encode action monitoring, not selection, during adaptive decision-making. J Neurosci 39:6668-6683.

Mars RB, Jbabdi S, Sallet J, O’Reilly JX, Croxson PL, Olivier E, Noonan MP, Bergmann C, Mitchell AS, Baxter MG, Behrens TE, Johansen-Berg H, Tomassini V, Miller KL, Rushworth MF (2011) Diffusion-weighted imaging tractography-based parcellation of the human parietal cortex and comparison with human and macaque resting-state functional connectivity. J Neurosci 31:4087-4100.

Mason S, Premereur E, Pelekanos V, Emberton A, Honess P, Mitchell AS (2019) Effective chair training methods for neuroscience research involving rhesus macaques (Macaca mulatta). J Neurosci Methods 317:82-93.

McLaren DG, Kosmatka KJ, Oakes TR, Kroenke CD, Kohama SG, Matochik JA, Ingram DK, Johnson SC (2009) A population-average MRI-based atlas collection of the rhesus macaque. Neuroimage 45:52-59.

Meng Y, Payne C, Li L, Hu X, Zhang X, Bachevalier J (2014) Alterations of hippocampal projections in adult macaques with neonatal hippocampal lesions: a diffusion tensor imaging study. Neuroimage 102:828-837.

Metzler-Baddeley C, Jones DK, Belaroussi B, Aggleton JP, O'Sullivan MJ (2011) Frontotemporal connections in episodic memory and aging: a diffusion MRI tractography study. J Neurosci 31:13236-13245.

Metzler-Baddeley C, Hunt S, Jones DK, Leemans A, Aggleton JP, O'Sullivan MJ (2012) Temporal association tracts and the breakdown of episodic memory in mild cognitive impairment. Neurology 79:2233-2240.

Milham MP, Ai L, Koo B, Xu T, Amiez C, Balezeau F, Baxter MG, Blezer EL, Brochier T, Chen A, Croxson PL, Damatac CG, Dehaene S, Everling S, Fair DA, Fleysher L, Freiwald W, Froudist-Walsh S, Griffiths TD, Guedj C, et al. (2018) An open resource for non-human primate imaging. Neuron 100:61-74.e62.

Mitchell AS, Gaffan D (2008) The magnocellular mediodorsal thalamus is necessary for memory acquisition, but not retrieval. J Neurosci 28:258263.

Mitchell AS, Baxter MG, Gaffan D (2007) Dissociable performance on scene learning and strategy implementation after lesions to magnocellular mediodorsal thalamic nucleus. J Neurosci 27:11888-11895.

Mitchell AS, Browning PG, Wilson CR, Baxter MG, Gaffan D (2008) Dissociable roles for cortical and subcortical structures in memory retrieval and acquisition. J Neurosci 28:8387-8396.

Mitchell DJ, Bell AH, Buckley MJ, Mitchell AS, Sallet J, Duncan J (2016) A putative multiple-demand system in the macaque brain. J Neurosci 36:8574-8585

Mitchell AS, Czajkowski R, Zhang N, Jeffery K, Nelson AJ (2018) Retrosplenial cortex and its role in spatial cognition. Brain Neurosci Adv 2:2398212818757098.

Murray EA, Rudebeck PB (2018) Specializations for reward-guided decisionmaking in the primate ventral prefrontal cortex. Nat Rev Neurosci 19:404-417.

Murray EA, Wise SP (2010) What, if anything, can monkeys tell us about human amnesia when they can't say anything at all? Neuropsychologia 48:2385-2405
Noonan MP, Walton ME, Behrens TE, Sallet J, Buckley MJ, Rushworth MF (2010) Separate value comparison and learning mechanisms in macaque medial and lateral orbitofrontal cortex. Proc Natl Acad Sci USA 107:20547-20552.

Noonan MP, Sallet J, Mars RB, Neubert FX, O'Reilly JX, Andersson JL, Mitchell AS, Bell AH, Miller KL, Rushworth MF (2014) A neural circuit covarying with social hierarchy in macaques. PLoS Biol 12:e1001940.

O’Reilly JX, Croxson PL, Jbabdi S, Sallet J, Noonan MP, Mars RB, Browning PG, Wilson CR, Mitchell AS, Miller KL, Rushworth MF, Baxter MG (2013) Causal effect of disconnection lesions on interhemispheric functional connectivity in rhesus monkeys. Proc Natl Acad Sci USA 110:13982-13987.

Parker A, Gaffan D (1997) The effect of anterior thalamic and cingulate cortex lesions on object-in-place memory in monkeys. Neuropsychologia 35:1093-1102.

Pelekanos V, Mok RM, Joly O, Ainsworth M, Kyriazis D, Kelly MG, Bell AH, Kriegeskorte N (2020) Rapid event-related, BOLD fMRI, non-human primates (NHP): choose two out of three. Sci Rep 10:7485.

Petrides M (1985) Deficits on conditional associative-learning tasks after frontal- and temporal-lobe lesions in man. Neuropsychologia 23:601614.

Poletti CE, Creswell G (1977) Fornix system efferent projections in the squirrel monkey: an experimental degeneration study. J Comp Neurol 175:101-128.

Phillips JM, Fish LR, Kambi NA, Redinbaugh MJ, Mohanta S, Kecskemeti SR, Saalmann YB (2019) Topographic organization of connections between prefrontal cortex and mediodorsal thalamus: evidence for a general principle of indirect thalamic pathways between directly connected cortical areas. Neuroimage 189:832-846.

Rudebeck PH, Saunders RC, Lundgren DA, Murray EA (2017) Specialized representations of value in the orbital and ventrolateral prefrontal cortex: desirability versus availability of outcomes. Neuron 95:1208-1220.e1205.

Rudebeck SR, Scholz J, Millington R, Rohenkohl G, Johansen-Berg H, Lee AC (2009) Fornix microstructure correlates with recollection but not familiarity memory. J Neurosci 29:14987-14992.

Saleem KS, Logothetis NK (2006) A combined MRI and histology atlas of the rhesus monkey brain in stereotaxic coordinates, Ed 1. San Diego: Academic

Saunders RC, Aggleton JP (2007) Origin and topography of fibers contributing to the fornix in macaque monkeys. Hippocampus 17:396-411.

Schmahmann J, Pandya D (2006) Fibre pathways of the brain. Oxford: Oxford UP

Shamy JL, Carpenter DM, Fong SG, Murray EA, Tang CY, Hof PR, Rapp PR (2010) Alterations of white matter tracts following neurotoxic hippocampal lesions in macaque monkeys: a diffusion tensor imaging study. Hippocampus 20:906-910.

Smith SM, Nichols TE (2009) Threshold-free cluster enhancement: addressing problems of smoothing, threshold dependence and localisation in cluster inference. Neuroimage 44:83-98.

Smith SM, Jenkinson M, Woolrich MW, Beckmann CF, Behrens TE, Johansen-Berg H, Bannister PR, De Luca M, Drobnjak I, Flitney DE, Niazy RK, Saunders J, Vickers J, Zhang Y, De Stefano N, Brady JM, Matthews PM (2004) Advances in functional and structural MR image analysis and implementation as FSL. Neuroimage 23:S208-S219.

Smith SM, Jenkinson M, Johansen-Berg H, Rueckert D, Nichols TE, Mackay CE, Watkins KE, Ciccarelli O, Cader MZ, Matthews PM, Behrens TE (2006) Tract-based spatial statistics: voxelwise analysis of multi-subject diffusion data. Neuroimage 31:1487-1505.

Sterpenich V, Albouy G, Darsaud A, Schmidt C, Vandewalle G, Vu TT, Desseilles M, Phillips C, Degueldre C, Balteau E, Collette F, Luxen A, Maquet P (2009) Sleep promotes the neural reorganization of remote emotional memory. J Neurosci 29:5143-5152.

Takashima A, Petersson KM, Rutters F, Tendolkar I, Jensen O, Zwarts MJ, McNaughton BL, Fernández G (2006) Declarative memory consolidation in humans: a prospective functional magnetic resonance imaging study. Proc Natl Acad Sci USA 103:756-761.

Takashima A, Nieuwenhuis ILC, Rijpkema M, Petersson KM, Jensen O, Fernández G (2007) Memory trace stabilization leads to large-scale changes in the retrieval network: a functional MRI study on associative memory. Learn Mem 14:472-479. 
Thomas C, Avram A, Pierpaoli C, Baker C (2015) Diffusion MRI properties of the human uncinate fasciculus correlate with the ability to learn visual associations. Cortex 72:65-78.

Tsivilis D, Vann SD, Denby C, Roberts N, Mayes AR, Montaldi D, Aggleton JP (2008) A disproportionate role for the fornix and mammillary bodies in recall versus recognition memory. Nat Neurosci 11:834-842.

Ungerleider LG, Mishkin M (1982) Two cortical visual systems. In: Analysis of visual behavior (Ingle DJ, Goodale MA, Mansfield RJ, eds), pp 549586. Cambridge, MA: Massachusetts Institute of Technology.

Ungerleider LG, Gaffan D, Pelak VS (1989) Projections from inferior temporal cortex to prefrontal cortex via the uncinate fascicle in rhesus-monkeys. Exp Brain Res 76:473-484.

Van Essen DC (2002) Windows on the brain: the emerging role of atlases and databases in neuroscience. Curr Opin Neurobiol 12:574-579.

Van Essen DC, Drury HA, Dickson J, Harwell J, Hanlon D, Anderson CH (2001) An integrated software suite for surface-based analyses of cerebral cortex. J Am Med Inform Assoc 8:443-459.

Van Essen DC, Glasser MF, Dierker DL, Harwell J (2012) Cortical parcellations of the macaque monkey analyzed on surface-based atlases. Cereb Cortex 22:2227-2240.

Vincent JL, Patel GH, Fox MD, Snyder AZ, Baker JT, Van Essen DC, Zempel JM, Snyder LH, Corbetta M, Raichle ME (2007) Intrinsic functional architecture in the anaesthetized monkey brain. Nature 447:83-86.

Von Der Heide RJ, Skipper LM, Klobusicky E, Olson IR (2013) Dissecting the uncinate fasciculus: disorders, controversies and a hypothesis. Brain $136: 1692-1707$
Wang JX, Kurth-Nelson Z, Kumaran D, Tirumala D, Soyer H, Leibo JZ, Hassabis D, Botvinick M (2018) Prefrontal cortex as a meta-reinforcement learning system. Nat Neurosci 21:860-868

Wang Z, Dai Z, Shu H, Liu D, Guo Q, He Y, Zhang Z (2017) Cortical thickness and microstructural white matter changes detect amnestic mild cognitive impairment. J Alzheimers Dis 56:415-428.

Wilson RC, Takahashi YK, Schoenbaum G, Niv Y (2014) Orbitofrontal cortex as a cognitive map of task space. Neuron 81:267-279.

Winkler AM, Ridgway GR, Webster MA, Smith SM, Nichols TE (2014) Permutation inference for the general linear model. Neuroimage 92:381397

Xiao D, Zikopoulos B, Barbas H (2009) Laminar and modular organization of prefrontal projections to multiple thalamic nuclei. Neuroscience 161:1067-1081.

Yamashita KI, Hirose S, Kunimatsu A, Aoki S, Chikazoe J, Jimura K, Masutani Y, Abe O, Ohtomo K, Miyashita Y, Konishi S (2009) Formation of long-term memory representation in human temporal cortex related to pictorial paired associates. J Neurosci 29:10335-10340.

Zakszewski E, Adluru N, Tromp DP, Kalin N, Alexander AL (2014) A diffusion-tensor-based white matter atlas for rhesus macaques. PLoS One 9: e107398.

Zhuang L, Sachdev PS, Trollor JN, Reppermund S, Kochan NA, Brodaty H, Wen W (2013) Microstructural white matter changes, not hippocampal atrophy, detect early amnestic mild cognitive impairment. PLoS One 8: e58887. 